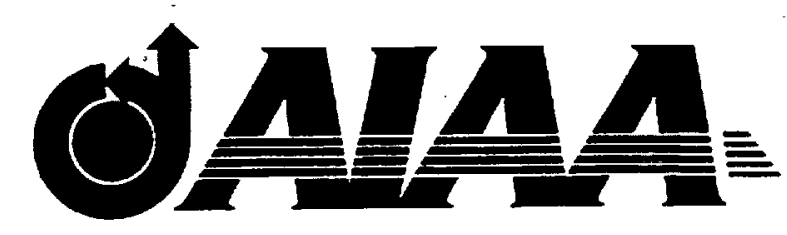

\title{
AIAA 2000-2099
}

\section{Investigation of Density Fluctuations in Supersonic Free Jets And Correlation with Generated Noise}

J. Panda

Modern Technologies Corporation, Middleburg Ht., OH 44130

R. G. Seasholtz

NASA Glenn Research Center, Cleveland, OH 44135

\section{6th AIAA/CEAS Aeroacoustics Conference 12-14 June 2000 / Lahaina, Hawaii}




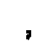

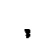

,

, 


\title{
Investigation of Density Fluctuations in Supersonic Free Jets And Correlation with Generated Noise
}

\author{
J. Panda* \\ Modern Technologies Corporation, Middleburg Hts., OH 44130 \\ R. G. Seasholtz \\ NASA Glenn Research Center, Cleveland, OH 44135
}

\begin{abstract}
The air density fluctuations in the plumes of fullyexpanded, unheated free jets were investigated experimentally using a Rayleigh scattering based technique. The point measuring technique used a continuous wave laser, fiber-optic transmission and photon counting electronics. The radial and centerline profiles of time-averaged density and root-mean-square density fluctuation provided a comparative description of jet growth. To measure density fluctuation spectra a two-Photomultiplier tube technique was used. Crosscorrelation between the two PMT signals significantly reduced electronic shot noise contribution. Turbulent density fluctuations occurring up to a Strouhal number (Sr) of 2.5 were resolved. A remarkable feature of density spectra, obtained from the same locations of jets in $0.5 \leq \mathrm{M} \leq 1.5$ range, is a constant Strouhal frequency for peak fluctuations. A detailed survey at Mach numbers $M=0.95,1.4$ and 1.8 showed that, in general, distribution of various Strouhal frequency fluctuations remained similar for the three jets. In spite of the similarity in the flow fluctuation the noise characteristics were found to be significantly different. Spark schlieren photographs and near field microphone measurements confirmed that the eddy Mach wave radiation was present in Mach 1.8 jet, and was absent in Mach 0.95 jet. To measure correlation between the flow and the far field sound pressure fluctuations, a microphone was kept at a distance of 50 diameters, $30^{\circ}$ to the flow direction, and the laser probe volume was moved from point to point in the flow. The density fluctuations in the peripheral shear layer of Mach 1.8 jet showed significant correlation up to the measurement limit of $\mathrm{Sr}=2.5$, while for Mach 0.95 jet no correlation was measured. Along the centerline measurable correlation was found from the end of the potential core and at the low frequency range $(\mathrm{Sr}<0.5)$. Usually the normalized correlation values increased with an increase of the jet Mach number. The experimental data point out eddy Mach waves as a strong source of sound generation in supersonic jets and fail to locate the primary noise mechanism in subsonic jets.
\end{abstract}

\section{INTRODUCTION}

The work presented in this paper addresses the issue of noise generation from supersonic free jets. The closing of the High Speed Civil Transport (HSCT) program has pointed out the technological advances needed in this arena for the success of a future supersonic, civilian aircraft. In addition, more stringent government and community requirements for aircraft noise is creating a further challenge to reduce noise emission from the current subsonic fleet. So far various experimental efforts depend on a "cut-and-try" approach, where one primarily looks for a change in the far field sound spectrum after making a new nozzle. A more sciencebased approach is to look for a change in the noise sources and therein lies the difficulty of the current experimental and computational techniques. In spite of the significant advances in the computational techniques, handling complex nozzle geometries and practical Reynolds numbers have remained a challenge. The theoretical background for noise emission by supersonic jets has been established in the past through the work of Lighthill (1954), Phillips (1960), Lilley (1972) and others. Although the recent DNS simulations (Freund 1998, Colonius et al. 1997) have successfully quantified the Lighthill stress tensor and the quadrupole sources, albeit for a simple geometry and very low Reynolds number, the same cannot be said of the experimental work. Direct measurement of these terms has remained an unattainable goal. Even at an elementary level, simple turbulence statistics for compressible flows are difficult to measure. Smits \& Dussauge (1996), in a recent book on turbulent shear layers, write: "The form of spectrum in compressible turbulence is still unknown." The present work at first takes on the task of measuring turbulence spectra, specifically density fluctuation spectra, using a Rayleigh scattering based technique. This is followed by a direct correlation study "between the cause and the effect (Lee \& Ribner, 1972)", that is, between the turbulent density fluctuation and far field noise. The goal is two fold: first, to provide a reliable and accurate database that can be used to validate computational aeroacoustics codes; second, to

\footnotetext{
* Resident research associate at NASA Glenn Research Center, Cleveland, $\mathrm{OH} 44135$. Corresponding author. Copyright $\bigcirc 2000$ by the American Institute of Aeronautics and Astronautics, Inc. All rights reserved.
} 
locate and determine the relative strength of various sound sources experimentally.

With the realization that large-scale vortical structures play a significant role in the development of free shear layers (Crow \& Champagne, 1971, Tam \& Burton 1984) a route different from the acoustic analogy approach (Lighthill 1954) began to emerge.

Experimental work on low Reynolds number jets by McLaughlin, Morrison \& Trout (1975) and Morrison \& McLaughlin (1979) provided sufficient proof that in supersonic jets large organized vortical structures are responsible for a significant part of sound generation. This approach is more 'physically realizable' and has been successful in predicting noise emitted from supersonic jets in the form of 'Mach wave' emission (Seiner, Bhat \& Ponton 1994). However, the noise field of subsonic jets and the high frequency part of the acoustic spectra could not be explained satisfactorily. The present work deals with both subsonic and supersonic jets. The experimental results were not strongly tied to any theory. It should be pointed out that the noise sources can be expressed solely in terms of density through the left hand side of the Lighthill's equation: $\partial^{2} \rho / \partial t^{2}-a_{0}^{2} \nabla^{2} \rho=\partial^{2} T_{i j} / \partial x_{i} \partial x_{j}$ (where $\rho$ is air density, $a_{0}$ is sound speed, and $T_{i j}$ is the turbulence stress tensor). The properties of large vortical structures, required in the instability wave based approach, can also be expressed through density $\rho(x, r, \varphi, t)=$ $\rho^{\prime \prime}\{r\} \exp [i\{\alpha x+n \varphi-\omega t\}]$ (where $\rho "$ is the eigenvector, $r, \varphi, x$ are cylindrical coordinates and $\alpha, n$ and $\omega$ are wavenumbers).

The lack of experimental data in compressible jets is primarily due to various problems in using traditional experimental tools. In the past, dynamic measurements in compressible jets were attempted using hot-wire probe or Laser Doppler Velocimetry (LDV). The success of using hot-wire by McLaughlin, Morrison \& Troutt (1975) and Morrison \& McLaughlin (1979) is attributed to the very low Reynolds number operation of their jet. At higher Reynolds numbers hot-wires are prone to breakage. At mixed subsonic and supersonic operating conditions the analysis of hot-wire signals become intractable. LDV, along with a high seeding rate, has been used (Lau 1981, Jiang \& Sislian 1998, among others) to determine velocity fluctuation statistics. However, various problems associated with seed particles following the flow, severe biasing problem in turbulent shear flows and difficulties in measuring spectra cast a doubt on such data. Similar problems associated with seed particles are encountered in Particle Image Velocimetry (PIV). In the present program a laser-based, non-intrusive Rayleigh scattering technique that depends on light scattering from air molecules is used. Since no seed particles are used, many problems associated with LDV and PIV can be overcome. The work of Wilson \& Damkevela (1970) needs special attention, as they measured mean-square density fluctuation using a non-intrusive crossbeam technique. The technique involves cross correlating deflections from two orthogonal laser beams passing through the jet. When the beams intersect, the crosscorrelation provides a mean square density fluctuation at the point of intersection. Some of their results are compared with similar data obtained from the present study. The crossbeam technique was also used to correlate density fluctuations to far field sound pressure fluctuations (Damkevala et al. 1973, among others). For this purpose, the beams were separated in space and information along the complete path of the beams was integrated. Moreover, the assumption of homogeneous turbulence, used in this technique, is invalid in a jet. All of these cast doubts on the validity of their results.

Based on Proudman's (1952) analysis, the above mentioned casuality approach of relating flow fluctuations to the far field noise was taken by many researchers. In this method, the velocity, density or pressure fluctuations in the jet were correlated with the sound pressure fluctuation measured by a microphone. The primary problem of all of these efforts is with the flow fluctuation measurement. The noise produced by intrusive hot-wire probe (Seiner \& Reethof, 1974) or microphone placed inside the flow (Meecham \& Hurdle, 1973, Hurdle, Meecham \& Hodder, 1974) contributed most of the correlation in some experiments. Later on LDV was used by Schaffer (1979) and Richarz (1979) among others. Schaffer wrote down the vast number of approximations and assumptions needed to relate the experimental data to theory. Nevertheless, an important issue of accuracy in velocity spectra measurements using LDV (Schaffer seeded only the primary jet not the entrained flow) was not answered. Before attempting to measure correlation, it is natural to expect that the individual components would be measured accurately. This important step, however, was skipped in all of the earlier efforts. A significant part of the present work concentrates on this aspect before attempting a correlation measurement. It is interesting to note Professor Ffowcs Williams' (1973) evaluation report on a host of experimental papers at the 1973 AGARD conference on Noise Mechanism: "Many papers presented at the Specialist Meeting dealt with different aspect of source location but none of them faced up to the difficult issue of interpreting or speculating on the degree of ambiguity that must inevitably be contained in the experimental results." A brief review of experimental techniques used for noise source locations can be found in Grosche (1979).

A part of the present work is an improvement and extension of our earlier effort to measure density fluctuation in supersonic jets (Panda and Seasholtz 1999b, henceforward referred to PS1). A modified optical setup and data reduction technique, presented in this paper, is 
found to minimize the effect of electronic shot noise in spectral information. Instead of measuring the scattered light by a single photo-multiplier tube (PMT), it is split into two parts and measured using two PMTs. Since the shot noise generated by individual detectors is uncorrelated, a cross-correlation between the two brought out the desired flow fluctuation information. The jet facility was also modified to suit flow-acoustics correlation measurements.

\section{EXPERIMENTAL SET-UP}

Experiments were performed at NASA Glenn Research Center using three different nozzles (one convergent and two convergent-divergent) operated at Mach numbers, $M$ $=0.95,1.4$ and 1.8 . The convergent-divergent nozzles were designed by the method of characteristics and their geometries were reported in Panda \& Seasholtz (1999b). All nozzles were $25.4 \mathrm{~mm}$ in exit diameter. The jet facility used a continuous supply of unheated compressed air. The facility was located in a large test chamber, which was not anechoic per se, but acoustic absorbent material was placed around the vicinity of the nozzle and in the ceiling and walls of the test cell to minimize reflection. Two $1 / 4$ inch microphones with the 'protection grid' removed were used to measure sound pressure fluctuation spectra. The coordinate system used throughout this paper is shown in fig. 1. The traversing system allowed flow measurements in the horizontal $x-y$ plane. The microphones were placed in the vertical $x-z$ plane to minimize the effect of reflection from the large optical lenses and beam traps. Whenever possible, various parts of the optical train, air supply duct and the jet facility were covered by $1 / 4$ inch thick polyurethane foam. However, the uncovered optical surfaces in the jet vicinity remained acoustic reflectors. The Rayleigh scattering system is somewhat elaborate and the following provides a description.

\section{A. Laser Rayleigh scattering:}

It is known that in Rayleigh scattering, the intensity of scattered light is proportional to the number density of gas molecules and in turn, is related to the local air density $(\rho)$. For the present experiment light intensity was measured using photomultiplier tubes and photon counting electronics. Therefore, the number of photoelectrons $(N)$ counted over a time interval $\Delta t$ can be expressed as:

$$
N=k \varrho \Delta t \text {, }
$$

where $\mathrm{k}$ is a proportionality constant to be determined through calibration. Figure 2 shows a schematic of the free air jet facility and the Rayleigh scattering setup. The Rayleigh scattering based point measurement technique used a continuous wave ( $\mathrm{CW}$ ) laser beam focused to a point in the flow, with the molecular scattered light collected and measured using PMTs. The green laser light (532-nanometer wavelength) from a CW, single frequency, frequency-doubled Neodymium Vanadate
(Nd: $\mathrm{YVO}_{4}$ ) laser was transferred to the jet vicinity by a $0.365 \mathrm{~mm}$ core diameter, multi-mode optical fiber and focused to a probe volume. Light scattered by the air molecules were collected at a $85^{\circ}$ scattering angle and focused on the face of another $0.55 \mathrm{~mm}$ core diameter multi-mode optical fiber. The combination of focusing and imaging optics makes the probe volume length of 1.03 $\mathrm{mm}$. The complete transmitting and receiving optics were mounted on a $x-y$ traverse that allowed the probe volume to be moved automatically over a plane. A somewhat more detailed description of the optical system can be found in Panda \& Seasholtz (1999c). The scattered light, collected by the receiving fiber, was transmitted to a quiet neighboring room away from the noisy environment. Here the collected light was collimated and then split into two equal part parts by a thin-film beam splitter. Each of the beams was refocused into individual photomultiplier tubes. Photon counting electronics were then used to measure light intensities. The advantage of the photon counting approach over the conventional measurement of analog PMT output (Pitts \& Kashiwagi 1984; Gouldin \& Halthore 1986; Graham, Grant \& Jones 1974) is a clearer estimate of measurement uncertainty due to electronic shot noise. The counting was performed over a series of contiguous time bins of specified interval. The maximum number of bins that can be used at a time was 16384 . Usually multiple sets of data were collected and passed on to a Personal Computer. The timer cycle for bin width was supplied externally through a programmable signal generator. All analog signals (from microphone amplifiers and pressure transducers indicating plenum and ambient conditions) were digitized and passed to the same computer. The data collection process was automated to move the laser probe volume from point to point in the flow field, perform the photon-counting process and collect the time history. When needed microphone and plenum transducer signals were also collected simultaneously.

The success of a Rayleigh scattering system depends on the use of clean, particle free air, minimization of stray scattered light and providing a stable, vibration free environment for some optical components. The last requirement is not as critical as for velocity and temperature measurement (where a Fabry-Perot Interferometer is used). Since, the facility was built to measure velocity and temperature in addition to air density, special arrangements were made to minimize the effect of loud jet noise. The primary jet was supplied with unheated, compressed air filtered to remove all dust particles. A clean co-flow through a coaxial nozzle of 200$\mathrm{mm}$ diameter surrounded the primary jet. An external air filter and air handling system were installed to produce this co-flow. The coaxial flow ensured dust-free air for the entrained flow. However a few particles were unavoidable. The second problem was stray reflected laser light. Due to 
the special orthogonal arrangement of the optical setup, the primary laser beam intersected the nozzle block when the probe volume was close to the nozzle exit. Between the nozzle exit and 1.3 diameter downstream of the exit the stray background light overwhelmed the Rayleigh signature and no measurement was possible. The third problem was condensation during the operation of Mach 1.8 nozzle. The primary supply air was dried to a dew point of $-50^{\circ}$ Celsius and moisture condensation was absent in the primary air jet. However, the entrained room air contained a significant amount of moisture, which condensed in the shear layer and caused significant stray scattering. An effective solution was found in operating the Mach 1.8 jet for a couple of hours continuously before starting the data acquisition. The jet facility was located in a closed test cell and a couple of hours of operation purged the moist room air and significantly reduced condensation.

\section{B. Density calibration}

A calibration process was necessary to determine the proportionality constant between the photon count rate and the air density, as well as to determine the residual stray light collected through the optics. The calibration was performed in the unheated plume of a convergent nozzle operated in the Mach number range of 0 to 0.99. At each operating condition the photon arrival rate was counted over a second duration and the jet density is calculated using isentropic relations. Subsequently, a straight line was fitted through the data to determine the proportionality constants $\mathrm{a}$ and $\mathrm{b}$.

$N=(a \varrho+b) \Delta t$

The additional constant $b$ is needed to account for the room light and stray scattered laser light. Figure 3 shows sample calibration curves. Since two counters were used, two sets of calibration constants $a_{1}, b_{1}$ and $a_{2}, b_{2}$ were calculated. The calibration was performed over a density range smaller than that encountered in the jet plumes. This is not of concern since the fundamental linear relationship between the molecular number density and intensity of scattered light holds true at any gas density.

\section{Time average density and root-mean-square density fluctuation measurement:}

The instantaneous flow density $\rho$ is divided into a timeaveraged part $\bar{\rho}$, and a fluctuating part $\rho^{\prime}$

$\varrho=\bar{e}+\varrho^{\prime}$

Photoelectron counting $\left(N_{i}, i=1,2, . . n\right)$ over a large number ( $n=65536$ ) of contiguous time bins covering more than 1 second time duration was used to measure the two parts. The mean density was related to the mean count rate $\mathrm{N}_{\mathrm{av}}$ by

$$
\bar{\varrho}=\frac{1}{a}\left(N_{a v}-b\right), \quad \text { where } N_{a v}=\frac{\Sigma N i}{n} \text {. }
$$

All density statistics presented here had to deal with the electronic shot noise arriving from the photo-multiplier tube. (No confusion should be made between 'jet noise' and 'shot noise'; the former is an acoustic phenomenon while the later is of photo-electronic in nature. Any optical intensity measurement is inherently affected by shot noise). Even when the incident light is of constant intensity (no density fluctuation), the rate of photoelectron emission by a PMT shows significant variation referred to as statistical photon count noise or 'shot-noise'. This noise is random in nature and follows Poisson's statistics. The density fluctuations cause the collected light intensity to vary and the joint statistics can be expressed through Mandel's Formula (Saleh \& Teich 1991). An important result of the Mandel's formula is that the variance of the photon count $\sigma_{N}^{2}$ is a sum of the variance of shot noise, $\sigma^{2}$ sh, and the variance of the light power fluctuation, $\sigma_{\mathrm{P}}{ }^{2}$ : $\sigma_{N}^{2}=\sigma_{s h}^{2}+\sigma_{P}^{2}$.

The variance of photon count was calculated from the measured data as:

$$
\sigma_{N}^{2}=\sum_{i=1}^{n} \frac{\left(N_{i}-N_{a v}\right)^{2}}{N_{i}-1}
$$

Another important result of Poisson's statistics is that the mean square of shot noise is equal to the time average of all counts:

$\sigma_{s h}^{2}=N_{a v}$

This allows for the light power fluctuation measurement: $\sigma_{\mathrm{P}}{ }^{2}=\sigma_{\mathrm{N}}{ }^{2}-\mathrm{N}_{\mathrm{av}}$. It is straightforward to show that the equivalent variance in the density fluctuation, $\bar{\rho}^{\prime 2}$, can be determined through the calibration constant $\mathrm{a}$, and the time width $\Delta t$, of the individual bins:

$\overline{\rho^{\prime 2}}=\frac{\sigma_{P}^{2}}{a^{2} \Delta t^{2}}$

The root-mean-square density fluctuation, $\rho_{\mathrm{rms}}$ was calculated as

$\varrho_{\text {ms }}=\sqrt{\overline{\rho^{\prime 2}}}$.

To reiterate, photon counting was performed over 65536 time bins. The average (equation 4 ) and mean square (equation 6) were calculated; the former was subtracted from the later, the mean square fluctuation was determined through equation 8 , and finally a square root of mean square provides ms density fluctuation.

The fundamental source of error in the time averaged density data is once again from the shot noise. The relative uncertainty in the measurement of $\mathrm{N}$ is determined through equation 7 as

$$
\frac{\sigma_{s h}}{N_{a v}}=N_{a v}{ }^{-\frac{1}{2}} \text {. }
$$


For the present experiment the count rate was high: between 8 and 12 million per second. Due to the small time width, the count accumulated in the individual bins was small, yet averaging over the large number of bins reduced the uncertainty to $<.05 \%$. Since, the contribution to uncertainty from the fundamental noise source was very low, that from a host of secondary sources became prominent. Occasional particles were unavoidable and their passage led to an increase in count. There were very fine oil droplets, perhaps picked up from the air compressor that caused a small difference in scattering intensity between the supply air and the cleaned ambient air. These secondary noise sources are difficult to quantify; the absolute density numbers are found to be repeatable within $\pm 1 \%$ of their quoted values.

The primary error source in ms data was due to a small deviation from Poisson's statistics (equation 6) in the PMT and photon counting electronics. Data obtained from quiescent clean air condition (steady light intensity) shows that the average count deviated slightly $( \pm 2 \%)$ from the mean square calculation. Since, the difference between the mean square and mean count was used to calculate the root-mean-square density fluctuation, the above deviation produced a positive bias error of about $4 \%$. The rms fluctuation measurement is susceptible to unsteadiness in the laser intensity, which fortunately, was very small. The second major source of error was due to the passage of occasional particles through the probe volume. As the probe volume was moved from close to the nozzle exit to 14 diameters downstream, the number of particles increased progressively from a few per second to the order of 100 per second. No measurements were performed beyond $\mathrm{x} / \mathrm{D}=14$. Large particles passing through the probe volume were easily detected by examining the bin to bin variation, and removed by neglecting counts above 5 times the rms value. This procedure, however, could not account for the small increases caused by particles passing through the vicinity of the beam waist. The bias error increased progressively from the nozzle exit to farther downstream as a larger number of particles were entrained into the jet. No data were taken beyond a downstream distance of 14D.

\section{Measurement of density fluctuation spectra}

The straightforward route to measure density fluctuation spectra is to take a one-sided Fourier transform of the sequence of photon counts $N(t)$,

$$
F_{\rho}(f)=\frac{2}{a \Delta t} \int_{0}^{\infty} N(t) e^{-i 2 \pi t} d t
$$

and then subtract a constant level that corresponds to the average shot noise contribution:
Shot noise floor $=$

$$
\frac{N_{a v}}{\text { no. of frequency bins in spectrum }}
$$

This was the route taken in the earlier effort (Panda \& Seasholtz, 1999). The method provided a crude estimate at the energetic low frequency part, and was unable to resolve the high frequency part of the spectrum. Shot-noise contributes to a fixed floor and a superimposed randomness to spectrum. The latter could not be removed through the subtraction process. In the present technique the collected light was split into two nearly equal parts and measured with two PMTs. The simultaneous photoelectron counting produced two series of data $N_{1}(t)$ and $N_{2}(t)$. The average counts from each of the time series were subtracted: $N_{1}(t)=N_{1}(t)-N_{1 a v}, N_{2}(t)=N_{2}(t)-N_{2 a v}$ and a cross-correlation,

$$
\overline{N_{1}^{\prime} N_{2}^{\prime}}(\tau)=\int_{-\infty}^{\infty} N_{1}^{\prime}(t) N_{2}^{\prime}(t+\tau) d t,
$$

removed the shot noise contribution for the most part. A cross spectral density function was used

$$
\begin{aligned}
F_{N_{1}^{\prime} N_{2}^{\prime}}(f) & =\int_{-\infty}^{\infty} \overline{N_{1}^{\prime} N_{2}^{\prime}}(\tau) e^{-i 2 \pi f \tau} d \tau \\
& =F\left(N_{1}^{\prime}\right) \cdot F^{*}\left(N_{2}^{\prime}\right)
\end{aligned}
$$

to calculate the density fluctuation spectra:

$$
F_{\rho^{\prime 2}}(f)=\frac{F\left(N_{1}^{\prime}\right) \cdot F^{*}\left(N_{2}^{\prime}\right)}{a_{1} a_{2}(\Delta t)^{2}}
$$

The $F$ indicates Fourier transform and $F^{*}$ complex conjugate of the transform. Usually a long record of either 262,144 (for higher Mach number jets) or 524,288 (low Mach number jets) data was made from multiple segments of 16384 data strings. The latter is the maximum number of contiguous counts delivered by the photon counters. Two such records were collected from two PMTs. The Welch method of modified Periodograms (1967) was used to calculate the cross-spectral density. Each long record was divided into small segments of 512 data points. The adjacent segments were overlapped by $50 \%$. The modified periodograms of corresponding segments from the two PMTs were calculated and then used to determine local estimates of cross-spectral density. All local estimates were averaged to obtain the final cross-spectral density. A source of experimental uncertainty in the spectral data is due to aliasing from the unresolved part of fluctuation spectrum. The photon counting process does not allow for an external anti-aliasing filter as used in the traditional signal analysis. However, the counting process sums up all fluctuations over the bin duration which acts as a special type of low pass filter. To illustrate, a 10 microseconds bin width effectively yields a sampling rate 
of $100 \mathrm{kHz}$. and the spectral information is resolved up to $50 \mathrm{kHz}$. A model of the summing up process shows that the contribution from fluctuations higher than the Nyquest range ( $50 \mathrm{kHz}$.) progressively falls down to zero at the sampling frequency $(100 \mathrm{kHz}$.). In addition, the low frequency, higher energy containing part of spectra is always resolved. The less energetic high frequency part does not affect measurements significantly. Therefore the aliasing error is expected to be small.

\section{E. Measurement of flow-sound cross correlation:} A $1 / 4$ inch diameter microphone was kept at a fixed position in the far field and the laser probe volume was moved from point to point in the flow field to determine correlation between the flow density fluctuations and sound pressure fluctuations. The microphone was kept at either $30^{\circ}$ or $90^{\circ}$ to the flow direction (Fig. 1) and at a radius of 50 nozzle exit diameters. The microphone signal was measured through an analog-to-digital (A/D) converter and the Rayleigh scattered light through a photon counting process. An external timer clock was used to time synchronize the acquisition processes. Towards this goal a signal generator was programmed to produce square waves of specified time period. The AN conversion occurred at each rising edge of the square waves, which also marked the beginning of individual time bins for photon counting. A time bin ended at the following rising edge. To verify time synchronization of this process, a synthetic signal was measured and compared. The synthetic signal was used to drive an electrostatic actuator and a photo-diode. The electrostatic actuator provided an excitation signal to the microphone diaphragm and the photo-diode, placed in front of the collection fiber, produced light intensity modulation. Satisfactory synchronization, observed over all frequency ranges tested, provided confidence in the data acquisition system.

The microphone was calibrated using a piston phone and the calibration constants were used to convert the voltage signal to instantaneous sound pressure fluctuation p'. The cross correlation density function between the flow density and sound pressure fluctuations are calculated using the sequence of photon counts and the microphone outputs:

$$
\begin{aligned}
& F_{N_{1}^{\prime} p^{\prime}}(f)=4 \int_{0}^{\infty} \int_{0}^{\infty} N_{1}^{\prime}(t) p^{\prime}(t+\tau) e^{-i 2 \tau t} d t d \tau . \\
& F_{e^{\prime} p}(f)=\frac{F_{N_{1}^{\prime} p^{\prime}}(f)}{a \Delta t}
\end{aligned}
$$

The cross-spectral density has real and imaginary parts,

$$
F_{\rho^{\prime} p^{\prime}}(f)=\operatorname{Re} F_{\rho^{\prime} p^{\prime}}(f)-i \operatorname{Im} F_{\rho^{\prime} p^{\prime}}(f) \text {, }
$$

which are used to determine the magnitude and coherence function.

$$
\begin{aligned}
& \left|F_{\varrho^{\prime} p^{\prime}}(f)\right|=\sqrt{\operatorname{Re}^{2} F_{\rho^{\prime} p^{\prime}}(f)+\operatorname{Im}^{2} F_{\rho^{\prime} p^{\prime}}(f)} \\
& \gamma_{\varrho^{\prime} p^{\prime}}^{2}(f)=\frac{\left|F_{N_{1}^{\prime} p^{\prime}}(f)\right|^{2}}{F_{N_{1}^{\prime} N_{1}^{\prime}}(f) F_{p^{\prime} p^{\prime}}(f)}
\end{aligned}
$$

Since the collected light was split and measured with two counters, the cross-correlation was performed two times: between microphone signal and either one of the two series of counts. Finally, an average of the two results was calculated. The cross-correlation minimizes shot noise in the photon count data, but a noise floor persists. To lower the noise floor more than half a million $(524,288)$ data points were collected for each data string. The Fourier transform used the segmenting and averaging process as outlined in the previous subsection. Unlike the calculations for density spectra, longer segments of the data string (4096) were used to account for the propagation time delay. The sound waves radiated from the flow fluctuations require finite time (distance of microphone/sound speed) to reach the far field microphone. Therefore, the time signature of the 'effect' (sound pressure fluctuation) is expected to lag behind the time signature of 'cause' (turbulent fluctuations). From a consideration of the sampling rate and microphone distance it was determined that for the present case only first few $(<5 \%)$ and last few data strings were affected by the time delay. By changing the length of data strings it was confirmed that the final correlation remained independent of this parameter.

\section{RESULTS AND DISCUSSION}

The choice of the three Mach number conditions $(0.95,1.4$ and 1.8) was deliberate. The purpose was to cover a range where both subsonic and eddy Mach wave radiation mechanisms produce jet noise. The schlieren photographs and the microphone spectra presented in Figure 4 shows that this goal has been attained. (In Fig. $4 \mathrm{a}$ a previously obtained schlieren photograph for Mach 0.99 jet is used.) It is known that in a supersonic jet, when turbulent eddies attain a speed faster than the ambient sound speed, a Mach wave emission process begins (Lowson \& Ollerhead 1968; Bishop, Ffowcs Williams \& Smith 1971; Papamoschou 1997) The Mach waves are ballistic shock waves attached to the supersonic eddies. The difference in the schlieren photographs for the three jets are primarily due to the inception of the eddy Mach wave emission process. Traces of these waves are visible in the photograph for the Mach 1.4 jet, while a stronger radiation pattern is visible in Mach1.8. There is a wide spread, between $0.89 \mathrm{Uj}$ to $0.6 \mathrm{Uj}$, where $U_{j}$ is the jet centerline velocity, in the convective velocity of turbulent eddies reported by various researchers. The estimated convective velocity (Table I) is always subsonic for Mach 0.95 jet; the Mach 1.4 jet is at the borderline, while eddies in Mach 1.8 jet are expected to 
attain supersonic speed. To determine the frequency of emission a microphone was kept in the near field at the position marked by $\otimes$ in the schlieren photographs. The respective microphone spectra are shown in Fig. 4(d). These spectra confirm the eddy Mach wave radiation for Mach1.4 \& 1.8 jets and their absence in Mach 0.95 jet. There is a common sharp hump at a lower Strouhal number $\left(\mathrm{Sr}=\mathrm{fD} / \mathrm{U}_{\mathrm{j}}, \mathrm{f}=\right.$ frequency in Hertz, $\mathrm{D}$ nozzle exit diameter, $U_{j}$ jet centerline velocity) around 0.2 in the near field spectra of fig. 4(d). The hump is associated with the large vortices present at the end of jet core. Zaman (1986) reported that the footprints of the vortices are seen along the entrained flow streamlines. Eddy Mach wave emission dominated the high frequency part of the sound spectra and peaked around $\mathrm{Sr}=1.5$. The far field sound pressure spectra are presented in Fig. 4(e). The $30^{\circ}$ angle was chosen to coincide with the peak sound radiation direction for cold jets as reported by Yu \& Dosanjh (1972). It should be pointed out that the ripples superimposed on the microphone spectra are telltale signs of reflection caused by large optical components placed around the jet. Nevertheless, the primary difference in spectra between the subsonic and supersonic conditions is seen as an increase in the overall level and a more energetic high frequency part. In a nutshell, Fig. 4 demonstrates that the three Mach number jets used in this experiment covered both subsonic and Mach wave sound emission regimes.

Before going into a discussion of density measurement, it is perhaps worthwhile to present some important aspects of viewing a jet flow through this parameter. In a low speed unheated air jet, the density variations are negligibly small. As the jet velocity is increased (or if heated) the density difference between the ambient air and the jet core increases. For the present unheated case increasing Mach number caused an increase in cooling which, in turn, increased the density of the primary air jet. The turbulent density fluctuations are caused by simple mixing between the ambient and primary jet fluid, as well as by the inertial effects (local acceleration and deceleration) of flow. The former perhaps contributes more to the density fluctuations; although, the inertial effects are expected to be the source of acoustic radiation. Table I shows the density difference present in the current experimental conditions. The accuracy of the current technique to measure density fluctuations improves as the density difference increases. Therefore, measurements made from higher Mach number conditions are expected to be more accurate. The experimental data are non-dimensionalized by the difference between the jet centerline and the ambient density, $\left(\rho_{j}-\rho_{2}\right)$. The timeaveraged data $(\bar{\rho})$ were non-dimensionalized as $(\bar{\rho}$ $\left.\rho_{\mathrm{a}}\right) /\left(\rho_{\mathrm{j}}-\rho_{\mathrm{a}}\right)$. Here $\rho_{\mathrm{j}}$ is the jet centerline jet density calculated from isentropic relations and $\rho_{a}$ is the ambient density. The parameter is unity at core and drops to 0 as the ambient condition is reached. The fluctuating density data were also normalized by $\left(\rho_{j}-\rho_{a}\right)$, i.e., $\rho_{\text {ms }} /\left(\rho_{j}-\rho_{a}\right)$. A minor disadvantage of this normalization is that the uncertainty in the finally presented data increases with a decrease of the jet Mach number. The same uncertainty of $\pm 1 \%$ in the absolute measurement of time-averaged density manifest as an error of $\pm 5 \%$ in the nondimesionalized presentation of data from Mach 0.95 jet and at $\pm 1.5 \%$ for Mach 1.8 jet.

\section{A. Time averaged data}

The centerline variation of time averaged density is shown in Fig. 5(a). The C-D nozzle surveys were performed at a pressure ratio that produced the weakest shocks in the core. It is to be pointed out that a completely shock free plume is never realized from the C-D nozzles at supersonic operating conditions. A literature survey of earlier data confirms this observation. Supersonic jets are wave-guides and a small manufacturing irregularity or even the shear layer turbulent fluctuations will produce standing waves in the form of weak shocks. A least shock operation point is determined by changing the operating pressure ratio and measuring the shock strength. For the present nozzles the least shock operation is measured to occur at $\mathrm{M}=1.395$ and 1.795 which are close to the design conditions of $\mathrm{M}=$ 1.4 and 1.8. The residual weak waves produce significant density modulations that persist far beyond the potential core (Fig. 5a). The inset schlieren photographs confirm the presence of the weak shocks. Downstream from the nozzle exit the spatial distance between the peaks and valleys reduces progressively, perhaps as a result of the lowering of local Mach number. For the present unheated jet, centerline density decays to the lower ambient value. Jiang \& Sislian (1998) used Rayleigh scattering to measure centerline decay of heated jets where density increased to the ambient value.

The root-mean-square density fluctuations of Fig. 5(b) shows that the turbulent fluctuations remain low over the first 6-10 nozzle diameters, then grow exponentially at the end of the potential core, and finally taper down slowly. As the potential core becomes longer with an increase of Mach number, the peak positions of the ms density fluctuation also moves further downstream. The peak fluctuations is about $0.22\left(\rho_{\mathrm{j}}-\rho_{\mathrm{a}}\right)$. Note that, similar to the time averaged data, the fluctuating quantities are also normalized by the difference between centerline and ambient density. The relative uncertainty level in the fluctuation data is higher due to the shot noise subtraction process described in the earlier section. In addition to a $4 \%$ bias error, there is a random uncertainty of $\pm 4 \%$. As mentioned earlier, the number of particles passing through the probe volume increased progressively and particularly affected data points obtained beyond the potential core. The measured data from this region are expected to be biased towards even higher values. The root-mean-square density fluctuations can be nearly zero 
under two situations. First if the turbulence fluctuations are very low, as in the potential core of the jet, and second if the flow velocity decays to the incompressible regime, such as in the far-field of jet development. In the later situation, there would be a significant velocity fluctuations while density fluctuations would be insignificant.

The jet spreading, due to the growth of shear layer, is shown in the time-averaged radial surveys of Fig. 6. The corresponding time-averaged velocity and temperature data, measured using spectrally resolved Rayleigh scattering technique, can be found in Panda \& Seasholtz, 1999a. The radial profiles demonstrate a progressive growth of the shear layer. The higher Mach number $M=1.8$ jet has the expected trend of a slower spreading rate compared to the Mach 0.95 case. The deviations from top-hat distribution, in the measured profiles from close to the nozzle exit, are due to the presence of the weak shock-cells. The radial profiles of root-mean-square density fluctuation are shown in Fig. 7. At the closest measurement station from the nozzle exit, the fluctuations peak in the shear layer around $y / D$ $= \pm 0.45$. The fluctuation profile at $x / D=2$ shows a fully turbulent shear layer with a quiescent core. The peak locations progressively approach the centerline, and onward from about 10D for Mach 0.95 jet and 12D for Mach 1.8 jet the centerline is the location for peak fluctuations. There is very little information on experimental measurement of scalar turbulent fluctuations in the available literature. One exception is the crossed-beam measurements of Wilson and Damkevala (1970). The cross-correlation between signals from two perpendicular, intersecting beams provided measurements of root-mean-square density fluctuations. In a Mach 0.6 jet they reported peak $\rho_{\text {rmd }}\left(\rho_{j}-\rho_{2}\right) \approx 0.2$ that agrees well with the current data.

By presuming that the ambient pressure is imposed everywhere in the jet plume (i.e., local pressure $p$ $\left.=p_{a m b}\right)$ it is possible to calculate time-averaged temperature from density. The inverse of the density profile becomes the temperature profile $\overline{\mathrm{T}}=\mathrm{p}_{\text {amb }} /(\bar{\rho} \mathrm{R})$, where $\mathrm{R}$ is the universal gas constant (Jiang \& Sislian, 1998). The above assumption may not be valid in a supersonic stream, where weak waves can produce pressure variation. It is also known that even in a subsonic jet local static pressure can deviate from the ambient pressure (Hussain \& Clark 1977, Zaman 1986). Therefore, no attempt is made in the present paper to calculate local temperature.

\section{Density fluctuation spectra}

Figure 8 shows the outcome and improvements attainable using 2 PMTs over single PMT. The width of time bins used in the photon-counting process was 10 microseconds. Following the Nyquest criterion, fluctuations up to $50 \mathrm{kHz}$. were resolved. Figure 8(a) shows spectra of the individual
PMT signals. Following equation 12 the dashed line shows the shot noise contribution. In Panda \& Seasholtz (1999b) the fluctuating part above the shot noise floor was presented. The improvement caused by cross-correlating the two PMT signals is shown in Fig. 8(b). The density fluctuation spectrum is better defined; the improvements are especially prominent at the high frequency end. Figures 8(c) and 8(d) show, respectively, the coherence and phase relation between the two PMT signals. The coherence function varies between 0 to 1 . A value of 0 would be measured if both signals were solely due to the electronic shot noise, i.e., there were no correlation between the two signals. A value of unity indicates perfect correlation. Therefore, the measured plot of the coherence function shows relative importance of the density fluctuation to the fluctuations from the electronic shot noise at various frequency ranges. Since, the same density fluctuations were measured by both PMTs, there should be no phase difference between the two signals. Figure 8(d) confirms this expectation and increases confidence on the measurement technique.

The cross-correlation process cannot eliminate noise from the density spectra; it lowers the noise floor. The finite number of data points used for the correlation and other electronic noise determine the final floor. An estimate of this noise floor is shown in Fig. 9 where the jet is turned off but the clean co-flow is present. Since there are no density fluctuations the spectral content is mostly due to the residual noise. The passage of occasional particles produces an additional contribution at the low frequency end (Seasholtz \& Panda 1999), seen as a small ramp in Fig. 9. The particular data set for Fig. 9 was obtained 10 diameters away from the nozzle exit where particle entrainment rate was relatively high. Spectra obtained at no flow condition and close to the nozzle exit do not show the low frequency contribution. Nevertheless, all density spectral description should be compared with the no-flow baseline and the part above the baseline is truly the desired spectrum. A comparison between Figs. 9 and 8(b) shows that at the high frequency end the measured fluctuations approach the residual noise floor. In addition to the noise base, the density spectra have a random uncertainty, the extent of which is visible in the randomness superimposed on the basic spectral shape of fig. 8(b).

The measurement technique was applied to determine the Mach number effect on density fluctuation spectra. For this purpose the laser probe volume was kept at a fixed position in the shear layer and the plume Mach number was varied between $0.5 \leq \mathrm{M} \leq 1.8$. The resultant spectra are shown in Fig. 10. When frequency values were normalized to Strouhal number an interesting fact emerged: the peak frequency of turbulence fluctuations was always found to occur at $\mathrm{Sr}=0.65$ at the particular measurement station. Hot-wire measurements at low Mach 
number jets, $M<0.5$, show a similar trend. The fact that this trend extends to supersonic conditions is shown for the first time. Another observation is that the spectra become fuller as Mach number is increased. The reason may not be completely due to a general increase of turbulence level, but instead may lie with an increase in the signal to noise ratio. As the Mach number is increased the difference of density between the ambient and the jet flow increases. The higher the difference, the better the signal to noise ratio. This in turn lifts a larger part of the spectrum above the noise floor.

Figure 11 presents a comparison between the density fluctuation spectra in Mach 0.95 and 1.8 jets. Parts (a) and (c) were measured along the peripheral shear layer and parts (b) and (d) were along the centerline. The data from the shear layer shows a gradual shift in the spectral peak from $\mathrm{Sr}=1.0$ to 0.2 with an increase in the downstream distance. The trend follows velocity fluctuation measurements in a low speed jet (Crow \& Champagne, 1971) and expectations from hydrodynamic stability analysis. The latter establishes that an increase in shear layer thickness with downstream distance leads to increased amplification of progressively longer wavelength and lower frequency Kelvin-Helmholtz instability waves. The spectra measured along the centerline (Figs. $11 \mathrm{~b}$ and $11 \mathrm{~d}$ ), however, differ from the velocity fluctuation measurement at low speed. Unlike the low speed measurements there is no definite hump at the passage frequency of large organized structures. All spectra peak at $\mathrm{Sr}=0$ for Mach 0.95 jet. For Mach 1.8 jet, weak humps are visible close to the nozzle exit, yet further downstream the peak occurs at $\mathrm{Sr}=0$. The primary observation of Figure 11, however, is the overall similarity in the spectral shapes, both along the centerline and the shear layer, between the two Mach numbers considered.

This similarity is further explored in fig. 12 where fluctuations occurring in the individual Strouhal frequencies are plotted from a large data set. The density fluctuation spectra were measured at a large number of points ( 8 to 12 axial by 10 radial points), and the meansquare fluctuations occurring at desired frequency bins were separated. For a uniform comparison, the square root of the mean-square fluctuations were non-dimensionalized by the difference of density between the jet core and the ambient density. In other words, from equation 15 the plotted variable can be written as:

$\frac{\rho_{s r}^{\prime}}{\rho_{j}-\rho_{a}}=\frac{\sqrt{F_{\rho^{\prime 2}}\left(f_{0}\right)}}{\rho_{j}-\rho_{a}}$,

where the frequency bin $f_{0}$ corresponds to the desired Strouhal number. The process is the same as applied to the earlier root-mean-square data. Figure 12 shows similarity in the distribution of turbulent fluctuations in the three Mach number jets considered. Fluctuations in the highest
Strouhal number occur close to the nozzle lip and in the shear layer. The position of the peak fluctuations progressively moves downstream as the lower $\mathrm{Sr}$ are considered. The $\mathrm{Sr}=0.25$ shows highest fluctuation at the end of the potential core and at $y / D \approx 0.3$. The fluctuations occurring at even lower Strouhal number, e.g. $\mathrm{Sr}=0.06$, peaks close to the centerline and further downstream. The primary change visible in the fluctuation pattern is an overall stretching with an increase in the Mach number. The slower growth of the instability waves and the resultant slower mixing process is the reason behind this stretching process. Returning to the sound pressure fluctuation data of Fig. 3, it is seen that the noise characteristics of the three Mach number jets are significantly different. This is in contrast with the similarity in the turbulent fluctuations. This led to the following correlation measurement between the flow fluctuations to the far field noise.

\section{Flow-sound correlation:}

The correlation data presented in this paper are in terms of normalized cross-spectral density in stead of the traditional cross-correlation coefficient. The primary advantage is the additional knowledge of frequency distribution that can be gained from such a representation. The intermediate steps towards the calculation of cross-spectral density between density fluctuations and sound pressure fluctuations are illustrated in Fig. 13. Part (a) shows the sound pressure fluctuation spectrum in a dimensional form ( $\mathrm{Pascal}^{2}$, no conversion to the common deciBel scale is performed). Part (b) shows the density spectrum measured using two PMTs. Part (c) presents the magnitude of the cross-spectral density function (equation 16). Finally, part (d) shows the coherence function obtained by normalizing cross-spectral density by the microphone spectrum and the spectrum of a single PMT output. The coherence function $\gamma$ provides a measure of linear dependency between the flow fluctuations and the sound fluctuations. All correlation data presented in the rest of the paper are in terms of $\gamma$. Note that the two PMT correlation process, used for density fluctuation measurement, was not used for flowsound correlation. Instead each of the PMT outputs was separately correlated with the microphone signal and an average between the two results was finally presented. Before proceeding further an estimation of noise floor in the calculations of coherence function is necessary. Similar to the density fluctuation data the crosscorrelation data are affected by electronic shot noise. The shot noise is independent of the microphone signal; therefore, the cross-correlation process is expected to remove it. However, a residual base level was always retained. To estimate this level, the jet was turned off (keeping the co-flow on) and the coherence function between the microphone and the PMT signals was measured. Figure 14 shows the base noise level obtained 
from this no-flow data. A comparison between the flow and no-flow data, respectively Figs. 13(d) and 14, shows that coherence function measured beyond $20 \mathrm{kHz}$ is completely due to the base noise level. However, for fluctuations slower than $20 \mathrm{kHz}$, the measured coherence is significantly above the noise base. In other words, flow fluctuations up to $20 \mathrm{kHz}$ are radiating noise in the far field. Since, a larger coherence also implies more efficient radiation process, density fluctuations occurring below $10 \mathrm{kHz}$ are more effective $(\gamma \approx 0.06)$ than fluctuations between $10-20 \mathrm{kHz}$. range, where coherence progressively falls to the noise floor $(\gamma \approx 0.002)$. In addition to the noise floor there is a convergence uncertainty that contributes towards the randomness seen in the coherence function plot.

A comparative study of correlation between turbulence fluctuations occurring along the jet centerline and sound pressure fluctuations at $\theta=30^{\circ}$ and $R=50 \mathrm{D}$ for the 3 Mach number jets is shown in Fig. 15. The microphone was kept fixed while the laser probe was moved from point to point along the centerline. Note that the ordinates are in a linear scale as opposed to the logarithmic scale used for Figs. 13 and 14. The individual plots are shifted and their new bases are marked by dotted lines. The no flow results at the bottom of each figure show the noise floor. In Mach 0.95 jet (Fig. 15a) no correlation was measured till the laser probe was moved beyond $x / D=6$. Fluctuations occurring at lower frequencies (up to $\mathrm{Sr}=0.2$ ) only are found to be correlated. As the jet Mach number was increased, higher frequency fluctuations, measured inside the potential core, began to radiate. For example, measurable coherence at $\mathrm{Sr}$ $=0.8$ was seen for Mach 1.8 jet (Fig. 15c). In addition, the levels were higher and a new trend in the peak correlation emerged. Similar to the density fluctuation spectra, the peak correlation progressively occurred at lower frequency as the laser probe was moved downstream. The peak coherence was around 0.1 and measured in the region $8 \leq$ $x / D \leq 12$.

The flow sound correlation was also measured using a microphone at $\theta=90^{\circ}$. This microphone was significantly more affected by acoustic reflection from the uncovered optical elements, beam dump and the uncovered part of the jet facility. In turn, the flow-sound correlation was also affected. Figure 16 presents some data obtained from Mach 1.8 jet using a $90^{\circ}$ microphone. The broad modulations in data seen especially in coherence plots from $x / D=12$ and 14 are believed to be due to acoustic reflection. A comparison with corresponding data from the $30^{\circ}$ microphone, Fig. 15(c), shows a significant decrease in the level and spread of correlation (note stretched ordinate for the $90^{\circ}$ case). Additional data measured for Mach 0.95 and 1.4 jets (not shown) showed an even larger decrease. In fact, no correlation was measured between the density fluctuations occurring anywhere in Mach 0.95 jet and the sound pressure fluctuations at the $90^{\circ}$ location.

The correlation data for fluctuations from the shear layer are presented in Fig. 17. The microphone was fixed at $\theta=30^{\circ}$ and the laser probe was moved along $y / D$ $=0.45$. A problem with the circumferential positioning of the laser probe needs to be mentioned. The shear layer is present all around the jet periphery and the correlation values are expected to be dependent on the circumferential position of the probe volume (Richarz, 1979). Referring to Fig. 2, the traversing arrangement allowed flow measurements only in the horizontal $x-y$ plane. Since the optical elements for the Rayleigh set up were also kept in this plane, acoustics reflections were at a maximum. To minimize reflections, microphones were always located in the vertical $x-z$ plane. The highest correlation is expected when both the flow fluctuations and sound pressure fluctuations are measured in the same $x-z$ plane. Therefore, the top part of shear layer needed to be accessed. Towards this end the complete nozzle facility was lowered by $0.45 \mathrm{D}$ : the radial position for the probe volume. The comparative study of Fig. 17 shows significant difference between the three Mach number conditions. The coherence function lies in the noise floor for all measurement stations in the subsonic Mach 0.95 jet; indicating no sound radiation from density fluctuations occurring in the shear layer. A very small rise above the noise floor is seen in Mach 1.4 jet, particularly at $x / D=6$ and 8 , indicating very weak radiation. Finally, Mach 1.8 jet shows significant rise above the noise floor, indicating a strong sound radiation that can be correlated with flow fluctuations happening at as high a frequency as the measurement limit: $\mathrm{Sr}=2.5$. When these shear layer data are compared with data measured along the centerline (Fig. 15), additional facts emerge. Only low frequency density fluctuations $(\mathrm{Sr}<0.8)$ along the centerline radiate noise while the high frequencies are primarily radiated from the shear layer. Note that the shear layer data are presented over a larger frequency range than the centerline data.

The implications of all of the above observations are not clear at the present time. It remains to be seen how various jet noise theories compare in predicting the above trends. This section on flow-sound correlation started with the observation that density fluctuation spectra in all three Mach number jets are similar. It is now clear that the same cannot be said of sound radiation to the far field, which explains the differences in the schlieren photographs and the far field acoustic spectra of Fig. 4. In Mach 0.95 jet only a small part of density fluctuation spectra $(\mathrm{Sr}<0.3)$ shows correlation with sound pressure fluctuations. The Mach 1.8 jet, on the other hand, shows strong correlation up to $\mathrm{Sr}=2.5$. The eddy Mach wave emission process is present in Mach 1.8 case and is absent in Mach 0.95 jet. The Mach 1.4 jet stays somewhat in between. For the 
Mach 1.8 jet the flow sound correlation follows the pattern of hydrodynamic instability waves. Close to the nozzle exit, the thin shear layer facilitates growth of the high frequency organized vortices and it is that part of density fluctuation which is found to radiate sound. Downstream from the nozzle exit the shear layer progressively thickens and lower frequency organized vortices become more energetic. The flow-sound correlation also follows suit. The 'eddies' in the eddy Mach wave emission are therefore nothing but the instability waves. Since, the instability waves are well predicted by the linear stability analysis, the success of this approach in predicting supersonic jet noise is now apparent (Tam \& Burton 1984).

The subsonic Mach 0.95 jet presents a different story. The same hydrodynamic instability waves are equally present in the subsonic jet, yet little correlation is measured between the flow and sound fluctuations. The density fluctuations occurring in the lip shear layer are uncorrelated with either the $90^{\circ}$ or $30^{\circ}$ microphone. Only weak correlation values are measured for fluctuations below $\mathrm{Sr}=0.3$ from the end of the potential core. The present technique fails to locate the primary noise source for subsonic jets. At this point, it should be pointed out that the normalized correlation coefficient (also expressed as coherence) shows the linear dependence between the flow fluctuations and radiated noise. Source (one frequency of flow fluctuations radiating a different frequency sound) and propagation non-linearity are not accounted for. It is possible that in a subsonic jets sound generation is primarily a non-linear process. The same arguments perhaps can be made for sound pressure fluctuations at $90^{\circ}$ to the jet axis; no correlation with the flow fluctuations are measured beyond $\mathrm{Sr}=0.5$ at any Mach number condition.

\section{SUMMARY AND CONCLUSION}

This paper presents an improved technique to measure air density fluctuation from Rayleigh scattered light, an application of this technique to map turbulent fluctuation spectra and finally relationship between the flow density fluctuations and the far field noise. The flows under study are unheated plumes of a subsonic Mach 0.95 and two supersonic, Mach 1.4 and 1.8 , jets.

The fundamental superiority of the Rayleigh scattering technique above the traditional hot-wire, Laser Doppler Velocimetry or Particle Image Velocimetry lies in its non-intrusiveness and its ability to obtain information directly from the gas molecules (no seed particles are used). A major problem of using the Rayleigh technique is in the weak scattering process. The light intensity measurements are significantly affected by electronic shot noise. A significant part of the paper is devoted to present the experimental technique, various means to reduce contribution from electronic shot noise, and uncertainty estimates. The Rayleigh scattered light from a point in the flow was measured using photo-multiplier tubes and photon counting electronics. The counting was performed on a large number of contiguous bins of small time duration. An average of all counts provided a measure of timeaveraged density. Subtraction of the average from the mean square provided measurement of mean-square density fluctuations. The spectral information of density fluctuations was gathered by dividing the collected light into two parts, measuring individual intensities using two sets of PMT and photon counting electronics and finally cross-correlating the two time signals. Since, electronic shot noise from the two PMTs is uncorrelated, the cross-correlation process significantly reduces its contribution. Finally, acoustic pressure signal from a microphone, placed in the far noise field, was correlated with the Rayleigh light to determine the portion of the density fluctuations radiating in the far field. A single PMT was sufficient to measure the Rayleigh signal as cross-correlation with the microphone signal reduced the shot-noise contribution. The noise floor, in all of the above measurements, was determined from no flow conditions (jet turned off).

Time averaged density measurements, obtained from radial and centerline surveys, confirmed expected trends such as a slow down of jet spread with an increase in Mach number. Nominally, $x / D=1.5$ was the closest position to the nozzle exit where a radial survey could be made. The root-mean-square fluctuation, normalized by the difference between jet and ambient density $\rho_{\text {rms }} /\left(\rho_{j}-\rho_{a}\right)$, was found to peak in the shear layer and fall to the noise floor in the quiescent core. The peak fluctuation was measured to be about 0.22 and was in agreement with previous measurements of Wilson \& Damkevala (1970). The centerline surveys showed the existence of weak shock-cell structures inside the pressure-matched supersonic jets. The time averaged schlieren photographs confirmed this observation.

The density fluctuation data presented in this paper are believed to be the first such measurements in supersonic jets. The sources of fluctuation are due to mixing between the jet and the ambient air, and inertial effects. The data provides a footprint of the turbulence fluctuations present in the jets. In order to compile a database for computational fluid dynamic simulations, density spectra were measured at a large number of points in the flow. When frequency values were normalized to Strouhal number, and spectral density values are divided by the difference of jet and air density, the fluctuations appeared remarkably similar in all three jets. Even the distribution of various Strouhal fluctuation components was similar. The primary difference was a spatial stretching with an increase in the Mach number. The distributions followed expected behavior of KelvinHelmholtz instability waves (Morris 1977). The high Strouhal frequency fluctuations were mostly energetic in 
the initial thin shear layer. As the shear layer thickened further downstream, the spectral peaks moved to lower Strouhal numbers.

Interestingly, the noise characteristics of the three jets, measured using a microphone and visible in spark schlieren photographs, were very different. The eddy Mach wave radiation process was present in the supersonic Mach 1.8 jet while it was absent in the subsonic Mach 0.95 jet; the Mach 1.4 case showed weak Mach waves. To determine the sound sources a 'causality approach' was followed where the density fluctuations were correlated with far field sound pressure fluctuations. The nonintrusive, particle free experimental technique provided more accurate and reliable measurements than any earlier effort. Most of the correlation measurements were performed with a microphone placed 50 diameters away and at the peak noise angle of $30^{\circ}$ to the jet axis. The data were expressed in normalized cross-spectral density (coherence) that provided frequency information. In Mach 1.8 jet when the laser probe was moved axially downstream along the lip shear layer $(y / D=0.45)$ the peak of flow-sound correlation is found to progressively move from the maximum resolved frequency of $\mathrm{Sr}=2.5$ to a lower $\mathrm{Sr}=0.5$. The peak coherence value also increased. A similar trend is seen for correlation from various points along the centerline. All of these point out that the KelvinHelmholtz instability waves were directly responsible for eddy Mach wave radiation. The scenario changed significantly for the subsonic Mach 0.95 case, the density fluctuations in the peripheral shear layer were found to be uncorrelated with the far field sound. Relatively weak correlation, only in the $0 \leq \mathrm{Sr} \leq 0.3$ range, was found from the end of the potential core and along the jet centerline. The same Kelvin-Helmholtz instability waves are present in the subsonic jets, yet the primary sound radiation mechanism is different and could not be identified in the present study.

\section{Acknowledgement}

The authors acknowledge fruitful discussions with Dr. Khairul Zaman and Dr. Milo Dahl of NASA Glenn Research Center.

\section{Reference}

Bishop, K. A., Ffowcs Williams, J. E. \& Smith, W. 1971 On the noise sources of the unsuppressed high-speed jet. J. Fluid Mech. 50(1) 21-31.

Colonius, T., Lele, S. K. \& Moin, P. 1997 Sound generation in a mixing layer. $J$. Fluid Mech., 330 375409.

Crow, S. C., \& Champagne, F. H. 1971 Orderly structure in jet turbulence. J. Fluid Mech., 48 547-591.

Damkevala, R. J., Grosche, F. R. \& Guest, S. H. 1973

Direct measurement of sound sources in air jets using the crossed beam correlation technique. $A G A R D C P 131$ on Noise Mechanisms, 3-1 to 3-16.

Ffowcs Williams, J. E. 1973 Technical evaluation report. AGARD CP 13 I on Noise Mechanisms, VII - XIX. Freund, J. B., 1998 Direct numerical simulation of the noise from a Mach 0.9 jet. FEDSM99-7251, $3^{\text {rd }}$ ASME/JSME Joint Fluid Engineering Conference. Graham, S. C., Grant, A. J. \& Jones, J. M. 1974 Transient molecular concentration measurements in turbulent flows using Rayleigh light scattering. ALAA J., 12(8) 1140-1142. Grosche, F. -R. 1973 Distribution of sound source intensities in subsonic and supersonic jets. $A G A R D C P$ 131 on Noise Mechanisms, 3-1 to 3-16.

Grosche, F. -R. 1979 How powerful are the experimental techniques of sound source location in aero-acoustics; what prospects are there for an improvement?

Mechanisms of Sound Generation in Flows, editor E. -A. Müller, Springer-Verlag.

Gouldin, F. C., \& Halthore, R. N. 1986 Rayleigh scattering for density measurements in premixed flames. Expts. in Fluids, 4 269-278.

Hurdle, P. M., Meecham, W. C. \& Hodder, K. 1974 Investigation of the aerodynamic noise generating region of a jet engine by means of the simple source fluid dilatation model. J. Acous. Soc. America, 56 1708-1721. Hussain, A. K. M. F. \& Clark, A. R. 1977 Upstream influence on the near field of a plane turbulent jet. Physics of Fluids, 20 1416-1426.

Jiang, L. Y. \& Sislian, J. P. 1998 Velocity and Density measurements in supersonic high-temperature exhaust plume. $A L A A J ., 36(7)$ 1216-1222.

dilatation model. AGARD CP131 On noise mechanisms. Lau, J. C. 1981 Effects of exit Mach number and temperature on mean flow and turbulence characteristics in round jets. J. Fluid Mech 105 193-218.

Lee, H. K., \& Ribner, H. S. 1972 Direct correlation of noise and flow of a jet. J. Acoustic. Soc. Of America. 52, no. 5 (pt. 1) 1280-1290.

Lighthill, M. J. 1954 On sound generated aerodynamically I. General theory. Proc. Royal Soc., A221 564-587.

Lilley, G. M. 1972 The generation and radiation of supersonic jet noise IV. Theory of turbulence generated noise. USAPL TR-72-53.

Lowson, M. V. and Ollerhead, J. B. 1968 Visualization of Noise from Cold Supersonic Jets. J. Acoustical Soc. Of America, 64(2) 624-630.

McLaughlin, D. K., Morrison, G. L. \& Troutt, T. R. 1975 Experiments on the instability waves in supersonic jets and their acoustic radiation. J. Fluid Mech 69 73-95.

Meecham, W. C. \& Hurdle, P. M. 1973 Use of crosscorrelation measurements to investigate noise generating region of a jet engine by means of the simple source fluid Moore, C. J. 1977 The role of shear-layer instability waves in jet exhaust noise. J. Fluid Mech. $80(2) 321-367$. 
Morris, P. J. 1977 Flow characteristics of the large scale wave-like structure of a supersonic round jet. J. Sound \& Vib., 53(2) 223-244.

Morrison, G. L. \& McLaughlin, D. K. 1979 Noise generated by instabilities in low Reynolds number supersonic jets. J. Sound \& Vib. 65(2) 177-191.

Panda, J., \& Seasholtz, R. G. 1999a Velocity and temperature measurement in supersonic free jets using spectrally resolved Rayleigh scattering. ALAA paper 990296.

Panda, J., \& Seasholtz, R. G. 1999b Density Fluctuation Measurement in Supersonic Fully Expanded Jets Using Rayleigh Scattering. ALAA paper 99-1870, presented at $5^{\text {th }}$ AIAA/CEAS Aeroacoustics Conference.

Panda, J. \& Seasholtz, R. J. 1999c Measurement of shock structure and shock-vortex interaction in underexpanded jets using Rayleigh scattering. Physics of Fluids, 11(12) 3761-3777.

Papamoschou, D. 1997 Mach wave elimination in supersonic jets, ALAA J., 35(10) 1604-1611.

Philips, O.M. 1960 On the generation of sound by supersonic turbulent shear layers. J. Fluid Mech 9(1) 1-28. Pitts, W. M., \& Kashiwagi, T. 1984 The application of laser-induced Rayleigh scattering to the study of turbulence mixing. J. Fluid Mech, 141 391-429.

Proudman, I. 1952 The generation of noise by isotropic turbulence. Proceedings of the Royal Society, A214 119132.

Richarz, W. G. 1979 Direct correlation of noise and flow of a jet using Laser Doppler. ALAA paper 79-057I, presented in $5^{\text {th }}$ Aeroacoustics Conference, Seattle, WA.

Saleh, B. E. A. \& Teich, M. C. 1991 Fundamentals of Photonics, John Wiley \& Sons, New York.
Schaffer, M. 1979 Direct measurements of the correlation between axial in-jet velocity fluctuations and far field noise near the axis of a cold jet. J. Sound \& Vib. 64(1) 7383.

Seasholtz, R. \& Panda, J. 1999 Multiple Point Dynamic Gas Density Measurements Using Molecular Rayleigh scattering, NASA TM 1999-209295.

Seiner, J. M. \& Reethof, G. 1974 On the distribution of source coherency in subsonic jets. ALAA paper 74-4.

Seiner, J. M., Bhat, T. R. S. \& Ponton, M. K. 1994 Mach wave emission from a High-Temperature Supersonic Jet. ALAA J. 32(12) 2345-2350.

Smits, J. A. \& Dussauge, J.-P. 1996 Turbulent shear layers in supersonic flow, AIP press, USA.

Tam, C. K. W. \& Burton, D. E. 1984 Sound generated by instability waves of supersonic flows. Part 2.

Axisymmetric Jets. J. Fluid Mech. 138 249-271.

Yu, J. C. \& Dosanjh, D. S. 1972 Noise field of a supersonic Mach 1.5 cold model jet, The Journal of the Acoustical Society of America, 51 no. 5 (part 1), 14001410.

Welch, P. D. 1967 The use of fast Fourier transform for the estimation of power spectra: A method based on time averaging over short, modified periodograms, IEEE Trans. on Audio and Electroacoustics, AU-15 70-73. Wilson, L. N. \& Damkevala, R. J. 1970 Statistical properties of turbulent density fluctuations. J. Fluid Mech 43(2) 291-303.

Zaman, K. B. M. Q. 1986 Flow field and near and far sound field of a subsonic jet, J. Sound \& Vib., 106(1) 116.
Operating conditions Table $\mathbf{I}$.

Sp. heat ratio, $\gamma=1.4$; Total temp., $T_{0}=300^{\circ} \mathrm{K}$; Ambient density, $\rho_{\mathrm{a}}=$ $1.16 \mathrm{Kg} / \mathrm{m}^{3}$, Ambient sound speed, $a=347 \mathrm{~m} / \mathrm{s}$

\begin{tabular}{llll}
\hline $\begin{array}{l}\text { Nozzle type } \\
\text { Minimum shock operation at }\end{array}$ & Convergent & $\mathrm{M} 1.4 \mathrm{CD}$ & $\mathrm{M} 1.8 \mathrm{CD}$ \\
$\mathrm{M}=$ & & 1.395 & 1.795 \\
Jet Velocity $\mathrm{Uj}(\mathrm{m} / \mathrm{s})$ & 316 & 411 & 486 \\
$\begin{array}{l}\text { Estimated eddy convection } \\
\text { speed } \mathrm{U}_{\mathrm{c}}(\mathrm{m} / \mathrm{s})=0.6 \mathrm{Uj}-0.89 \mathrm{Uj}\end{array}$ & $190-282$ & $247-366$ & $292-433$ \\
Jet density $\rho_{\mathrm{j}}\left(\mathrm{kg} / \mathrm{m}^{3}\right)$ & 1.36 & 1.6 & 1.89 \\
Difference $\left(\rho_{\mathrm{j}}-\rho_{\mathrm{a}}\right)\left(\mathrm{kg} / \mathrm{m}^{3}\right)$ & 0.2 & 0.44 & 0.73 \\
Frequency for $\mathrm{Sr}=1(\mathrm{kHz})$ & 12.4 & 16.2 & 19.1 \\
\hline
\end{tabular}




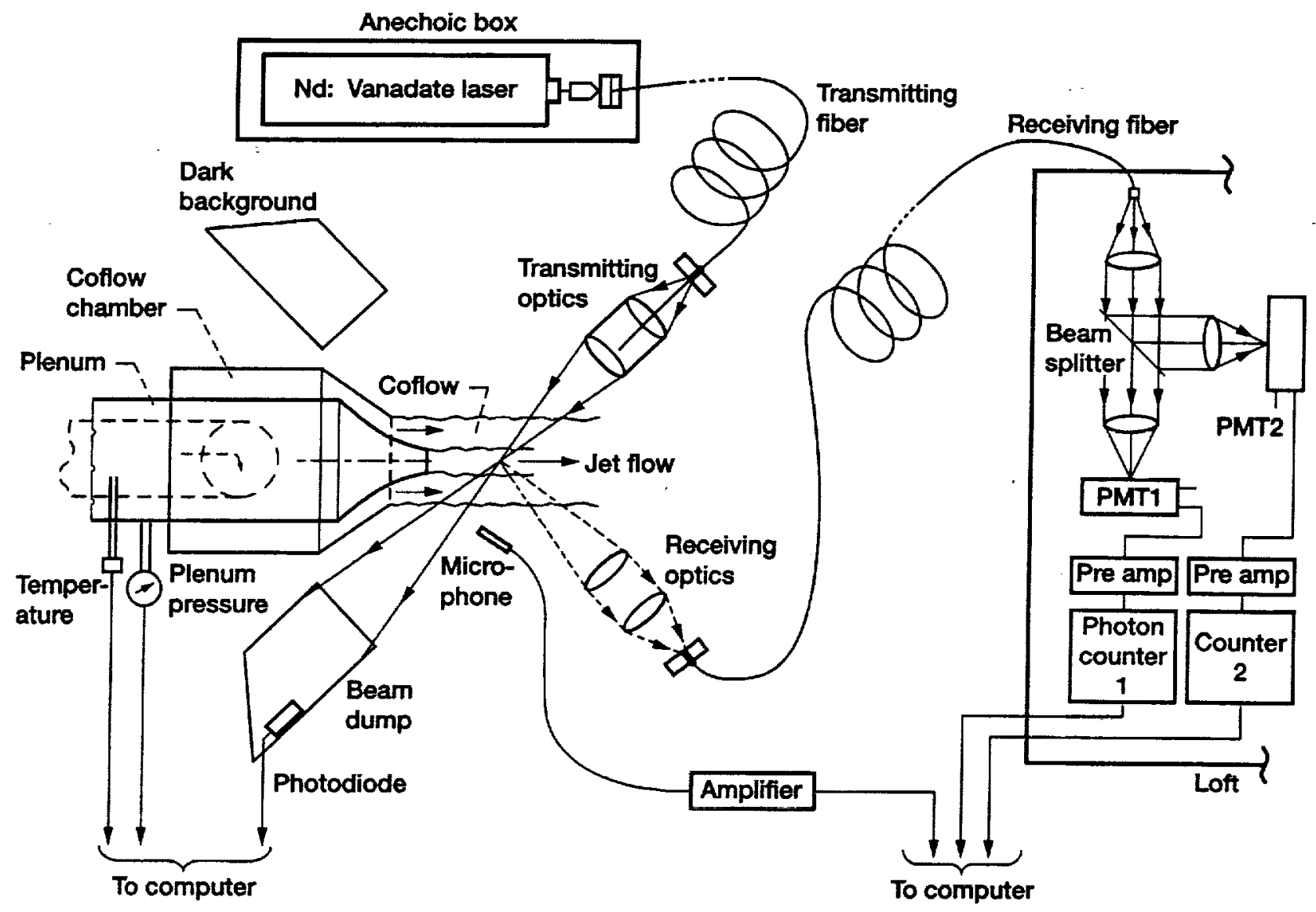

Fig 2. Schematic of jet facility and Rayleigh scattering setup.

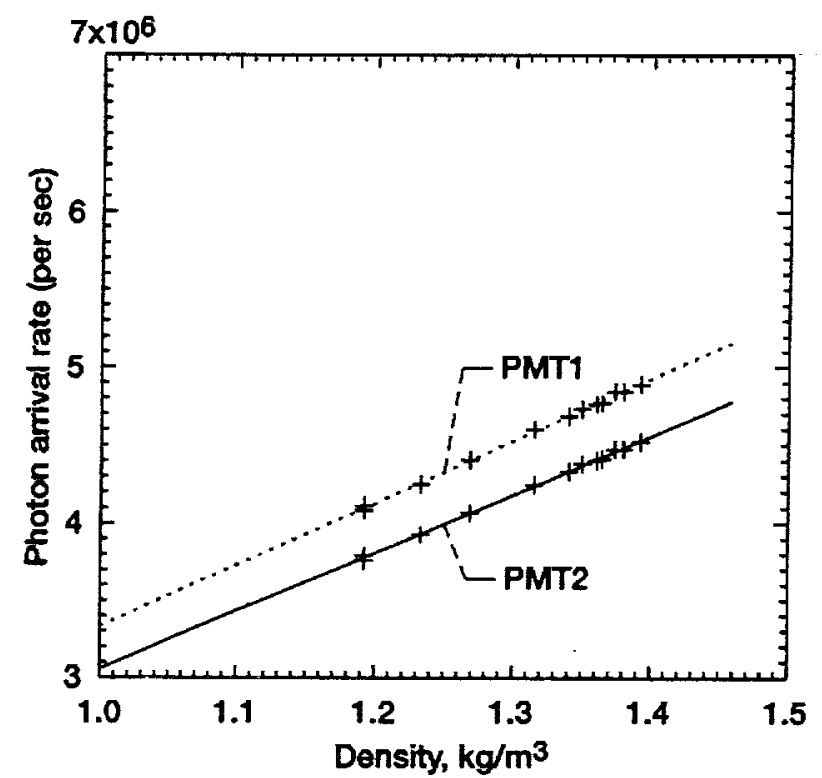

Fig 3. Calibration curve of Rayleigh scattered light to measure air density. Straight lines show least square fit. 

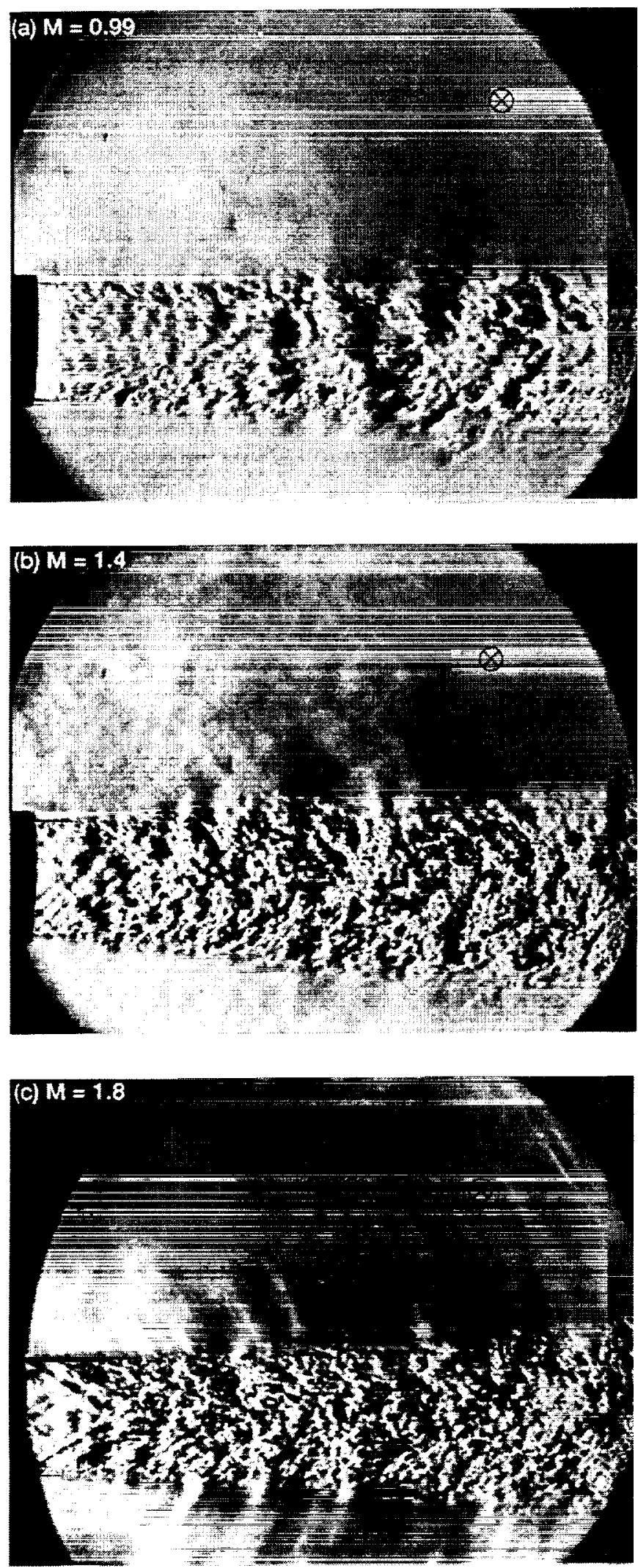

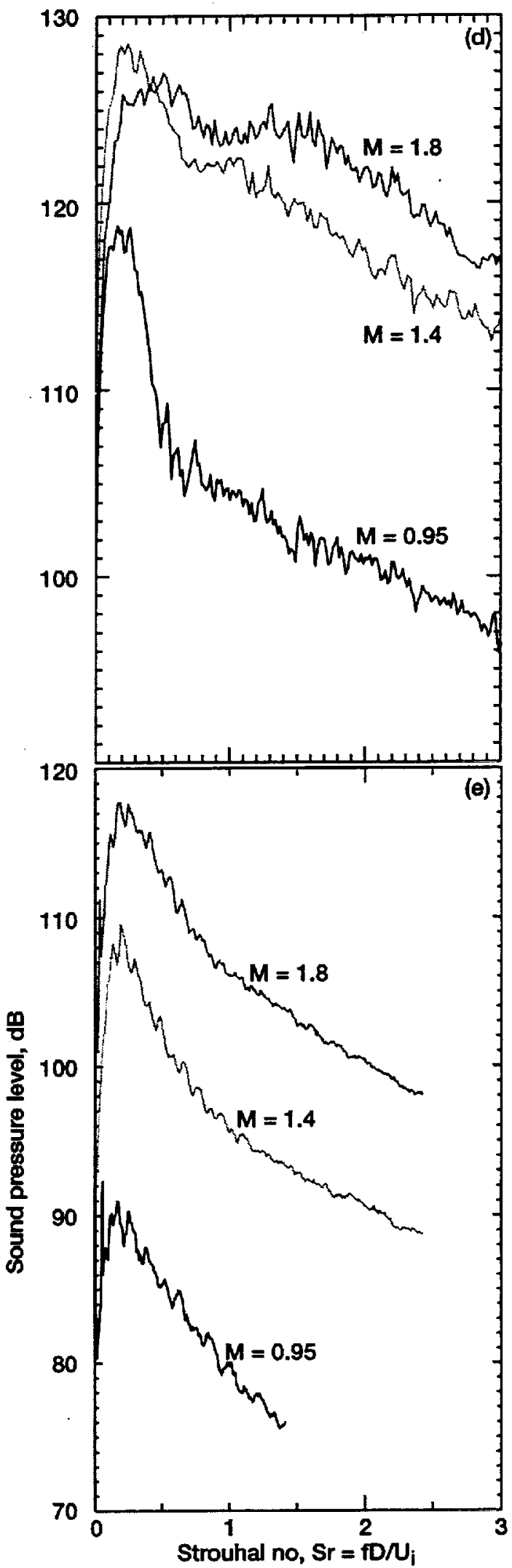

Fig 4. (a), (b), (c) Spark schlieren photograph of fully expanded jets at indicated Mach number conditions; (d) pressure fluctuation measured at $x / D=4, y / D=2$ (shown by $\otimes$ in schlieren photos); (e) sound pressure fluctuations at 50D and $30^{\circ}$ to the flow direction. 


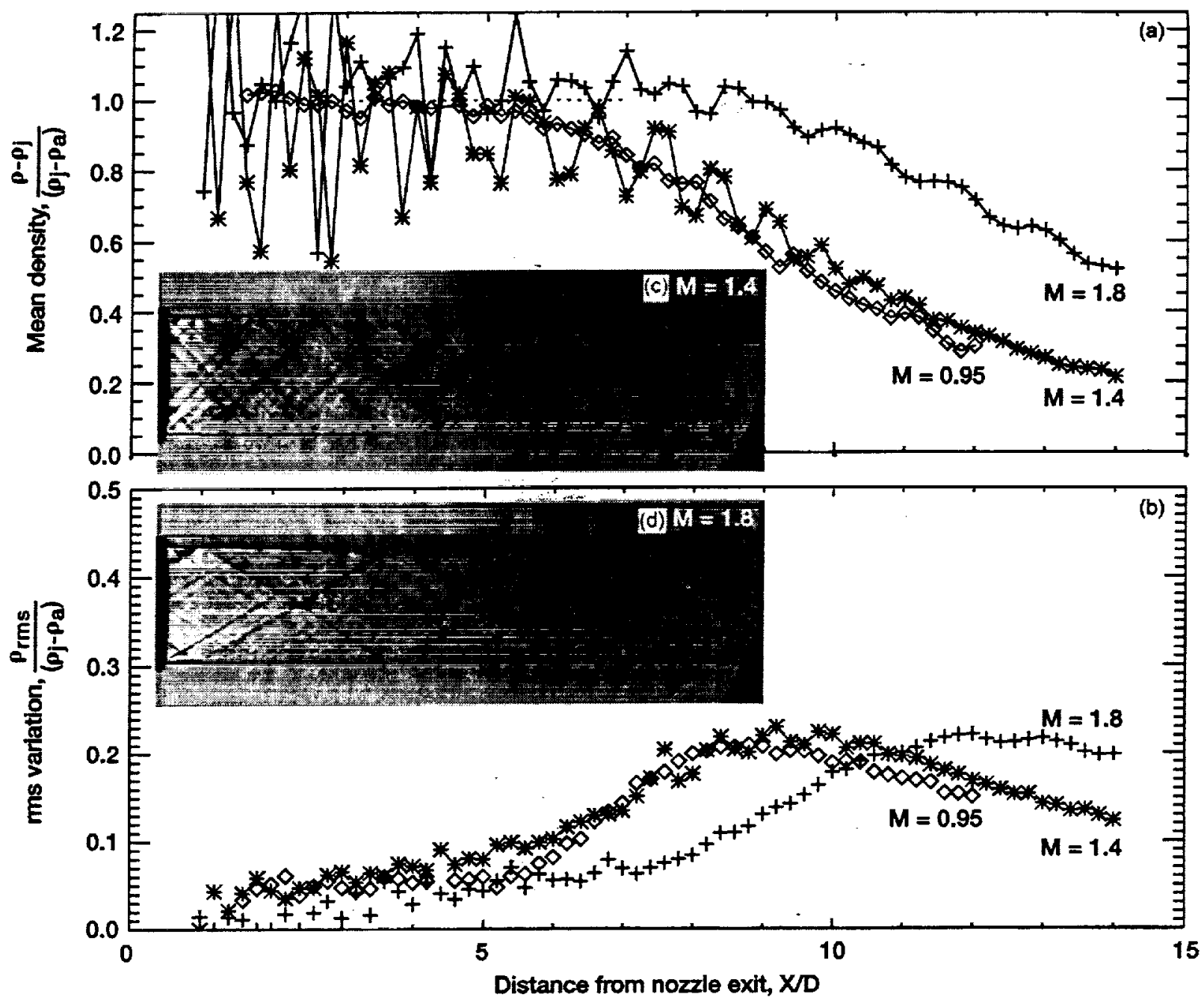

Fig 5. Centerline variations of (a) time-averaged density and (b) root-mean-square density fluctuations for indicated Mach no jets. The insets (c) and (d) are time averaged schlieren photographs. 


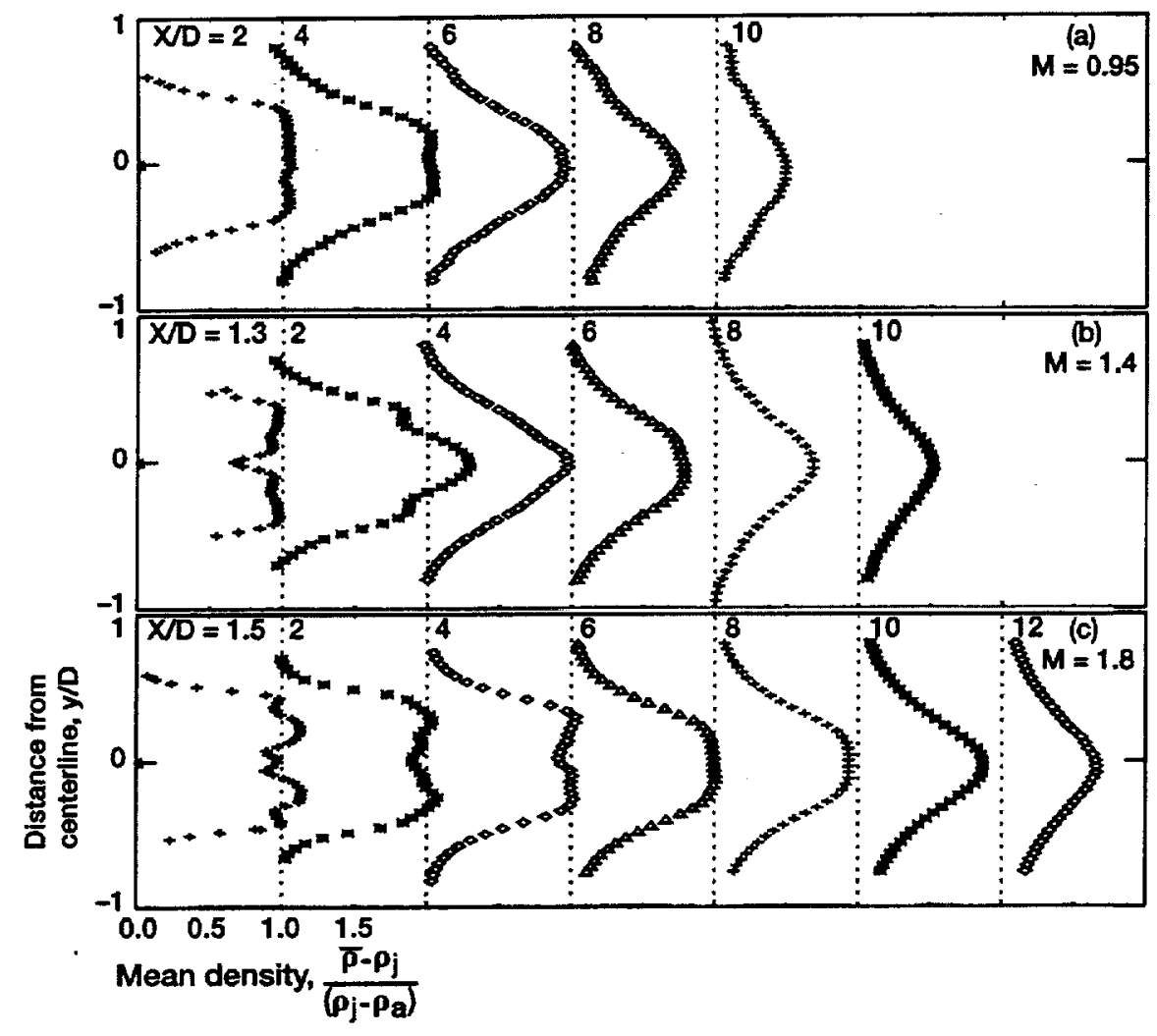

Fig 6. (a), (b), (c) Radial profiles of time-averaged density measured at indicated axial stations and Mach no conditions.

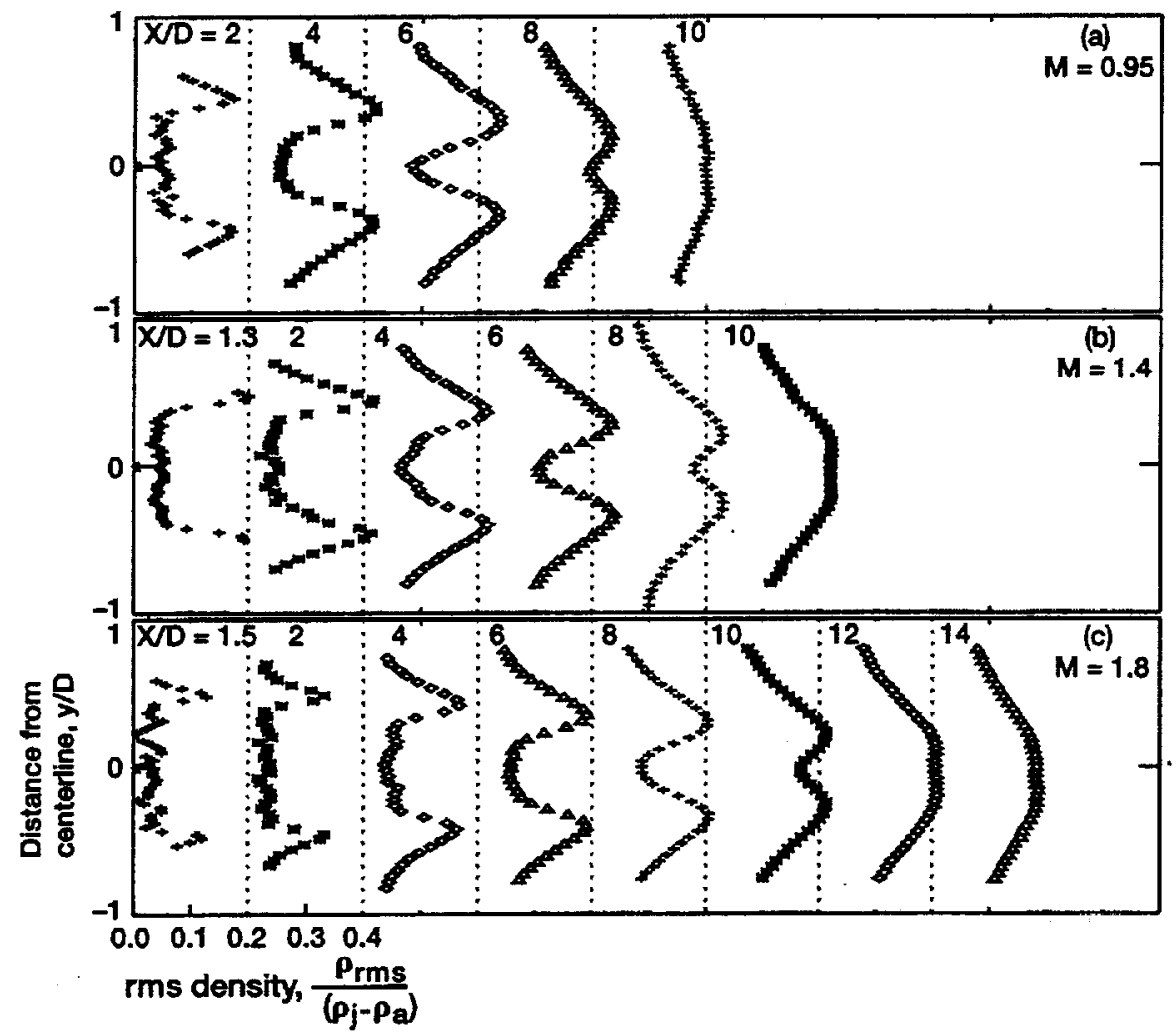

Fig 7. (a), (b), (c) Radial profiles of root-mean-square density fluctuations measured at indicated axial stations and Mach no conditions. 

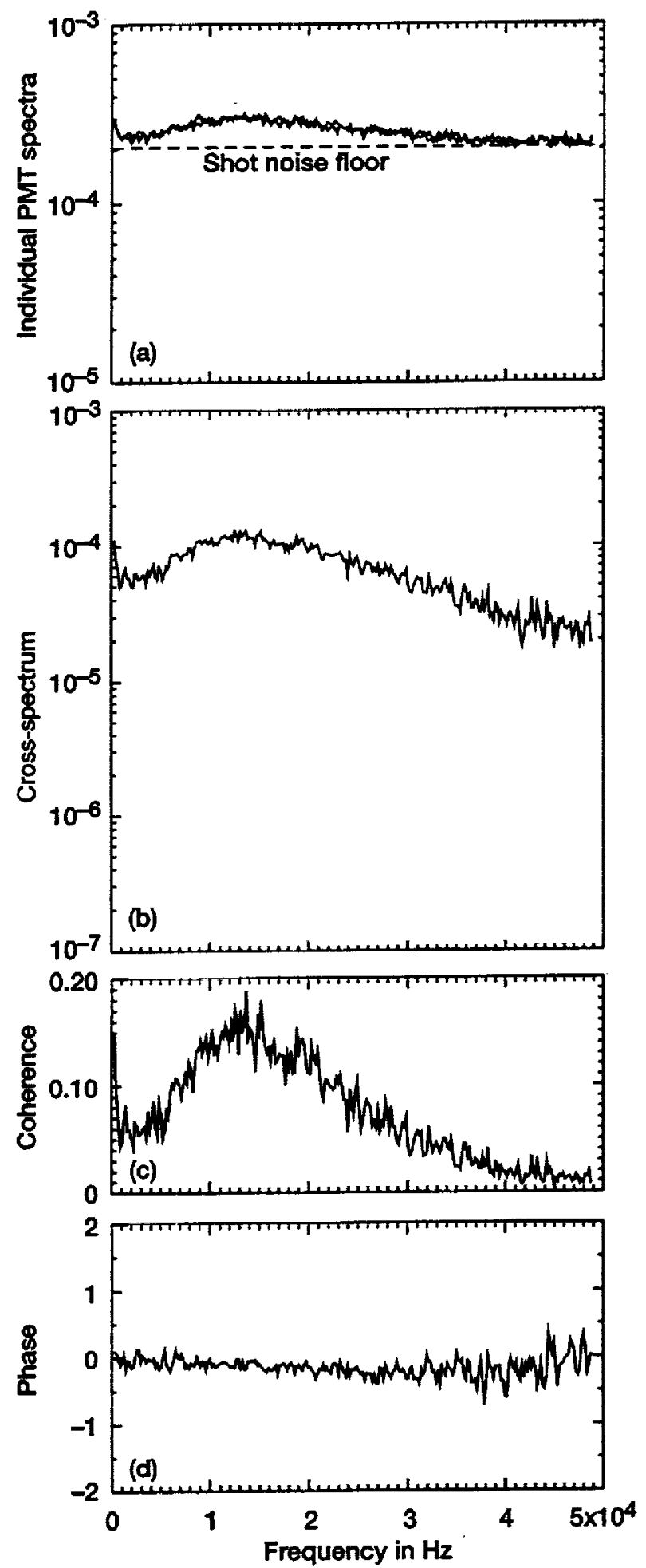

Fig 8. Cross-correlation between two PMTs to obtain density fluctuations spectrum. Data from Mach 1.8 jet at $y / D=0.48$ and $x / D=2.4$. (a) Spectra of individual PMT signals (b) cross-spectrum between the two providing density spectrum, (c) normalized crossspectrum (d) cross-spectral phase.

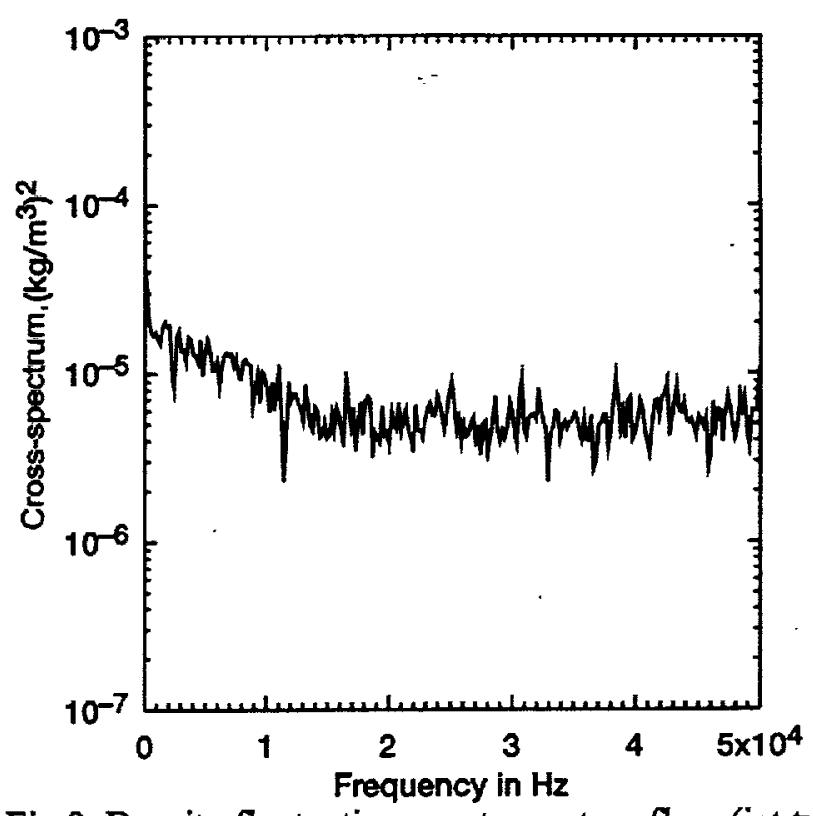

Fig 9. Density fluctuation spectrum at no flow (jet turned off) condition.

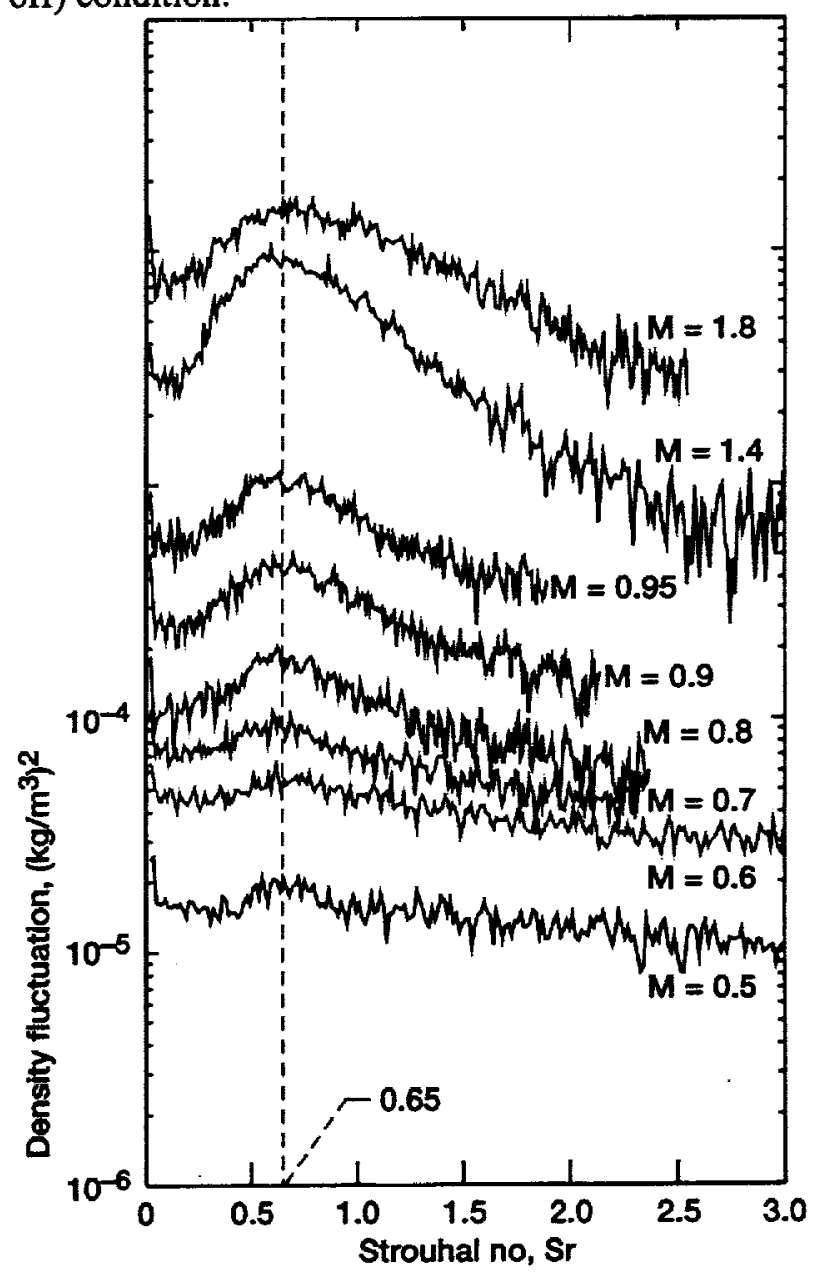

Fig 10. Density fluctuation spectra from a fixed position $(x / D=2.5, y / D=0.45)$ in the jet but at different Mach numbers. The vertical dashed line locates spectral peaks. Individual spectra are separated by a multiplication factor of 2 . 

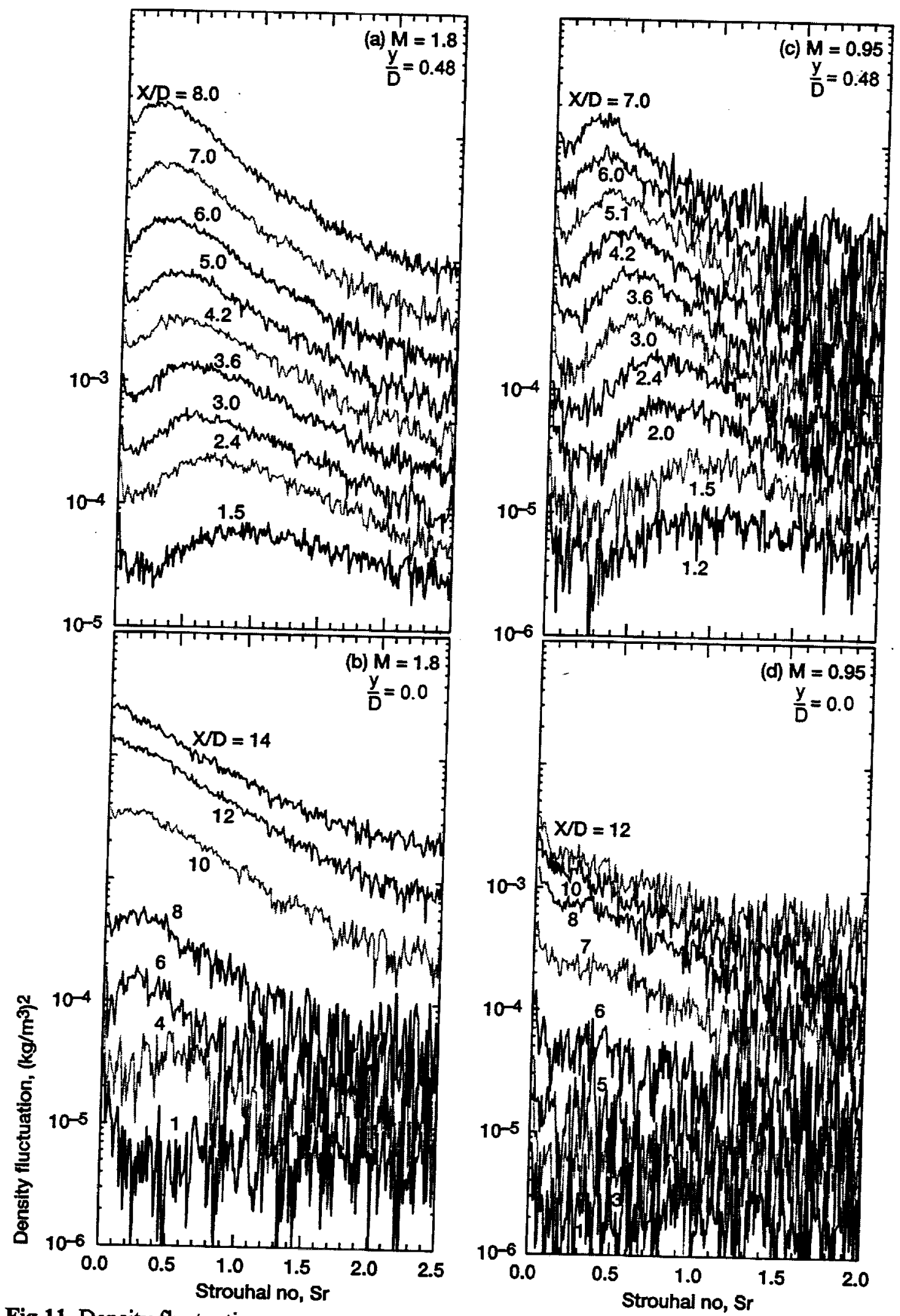

Fig 11. Density fluctuation spectra measured at indicated axial stations and along (a), (b) $y / D=0.48$ and (b), (d) centerline of Mach 0.95 and 1.8 jets. Individual spectra are separated by a multiplication factor of 2 . 


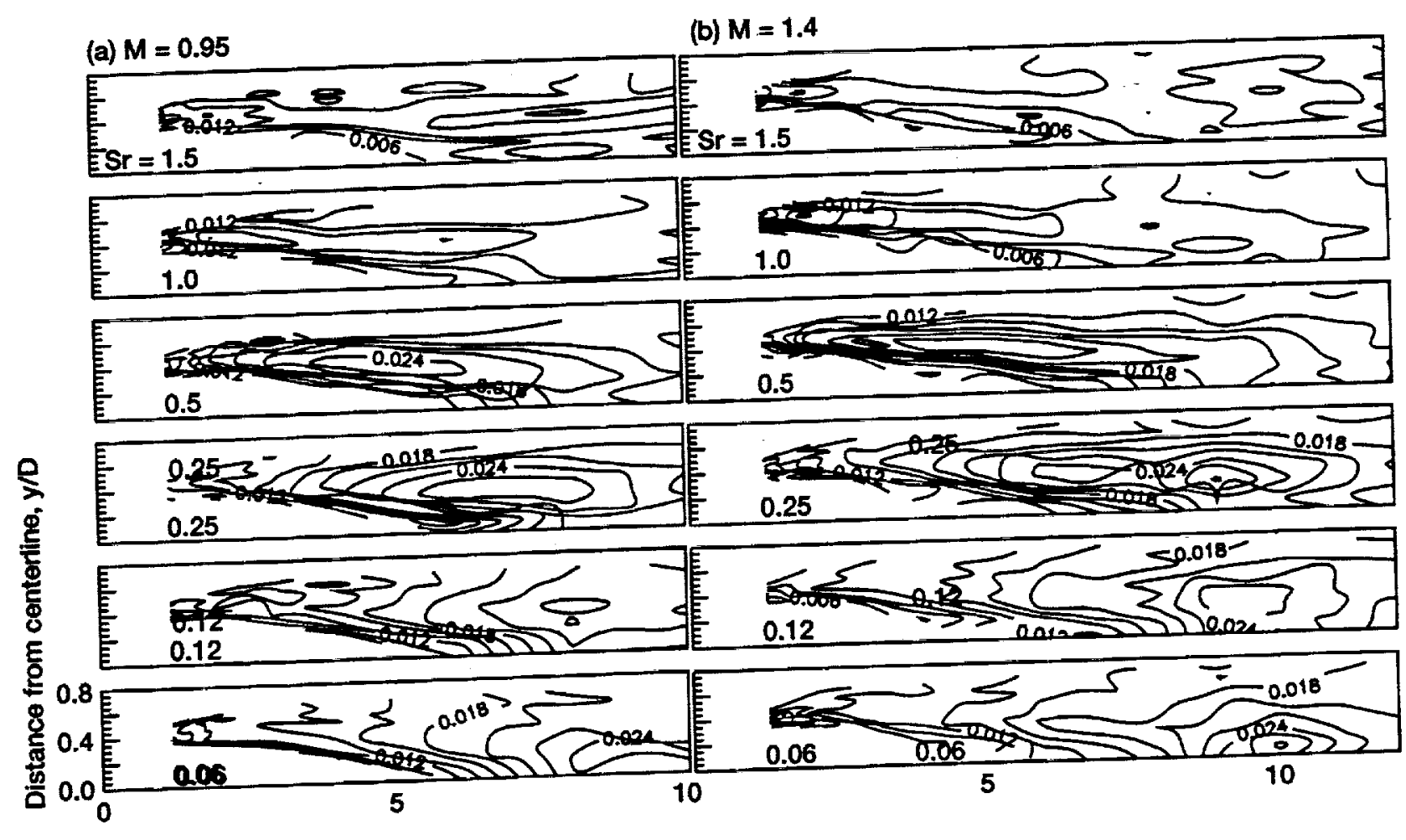

(c) $M=1.8$

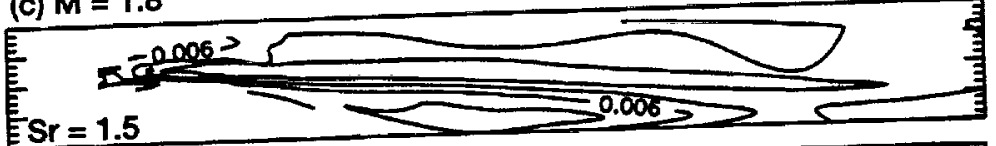

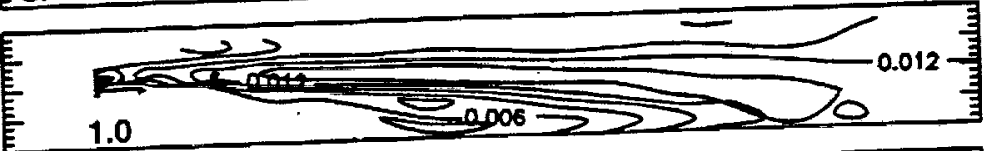
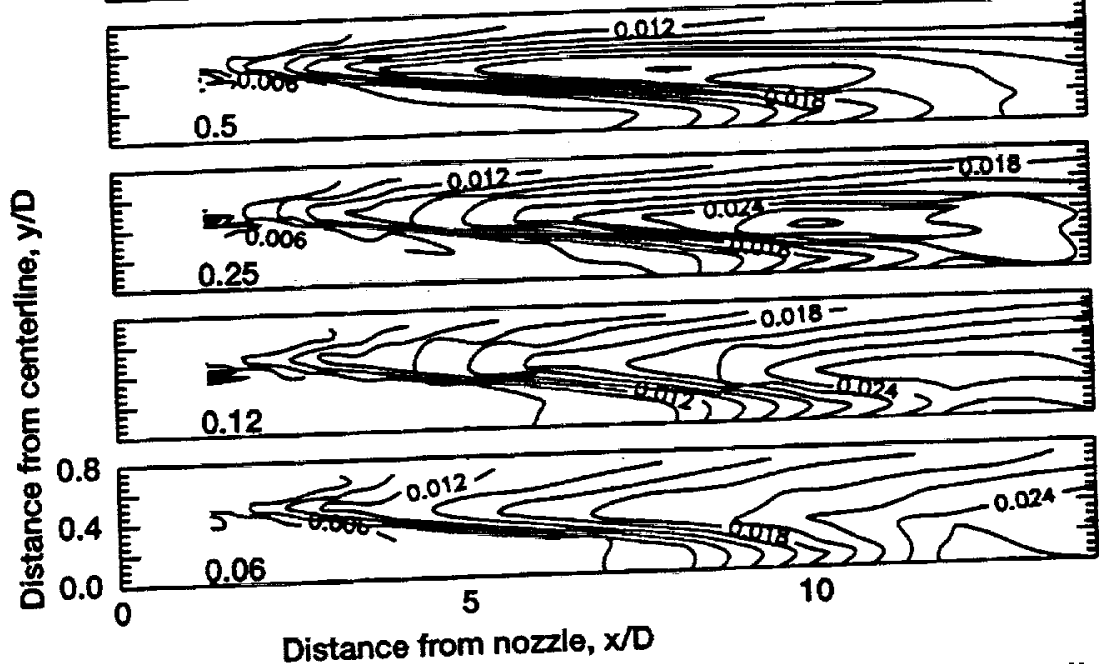

Distance from nozzle, $x / D$

Fig 12. The distribution of density fluctuations (normalized by $\rho_{j}-\rho_{a}$ ) at the indicated Strouhal frequencies for three different Mach numbers. Contours are at intervals of 0.002 . 

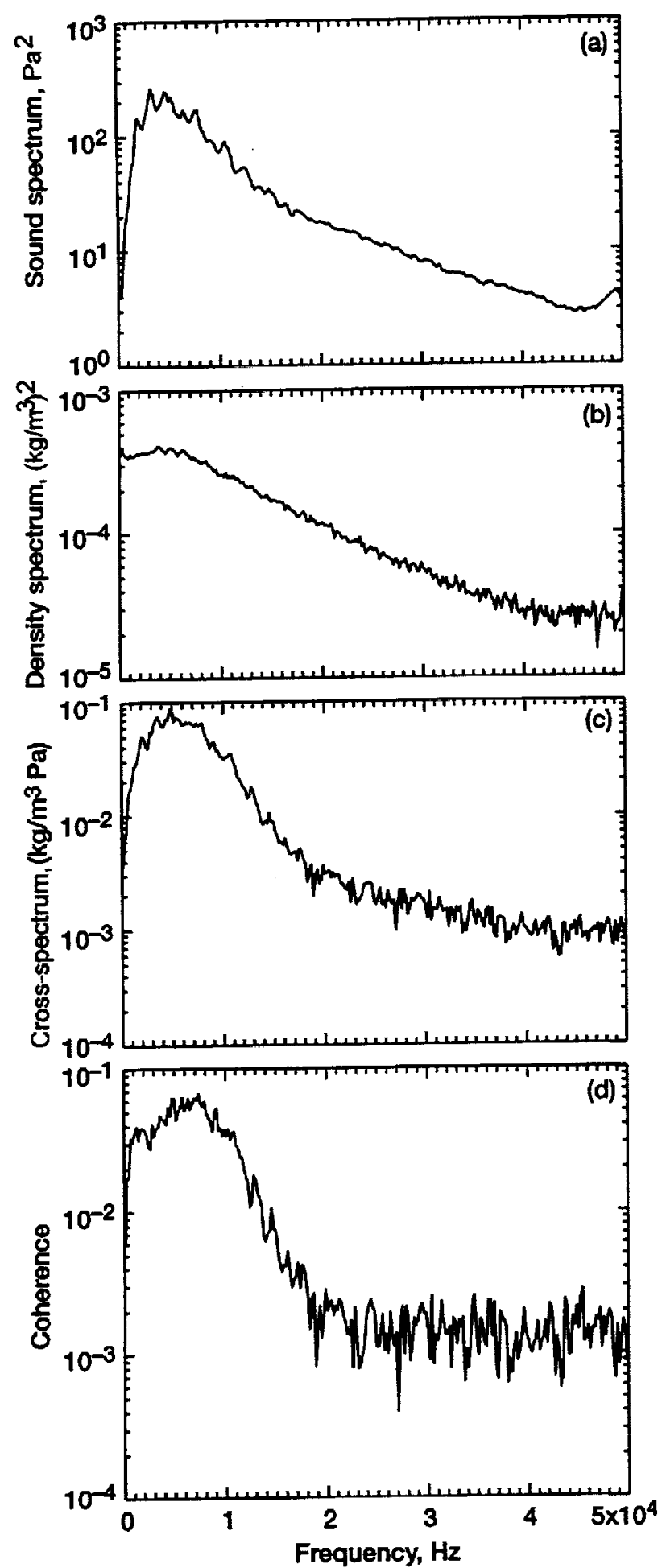

Fig 13. Cross-correlation between flow density fluctuations and sound pressure fluctuations in Mach 1.8 jet; laser probe at centerline and $\mathrm{x} / \mathrm{D}=10$, microphone at far field $x / D=50, \theta=30^{\circ}$. (a) Sound pressure spectrum, (b) density spectrum, (c) cross spectrum and (d) normalized cross spectrum (coherence).

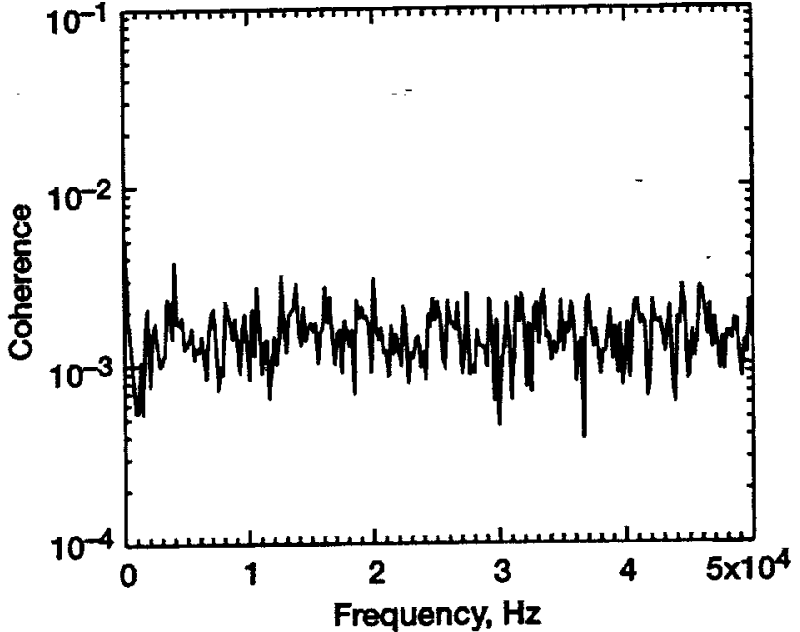

Fig 14. Normalized cross-spectrum between density and sound fluctuations at no flow condition (jet turned off). Laser and microphone positions are as in Fig. 13. 

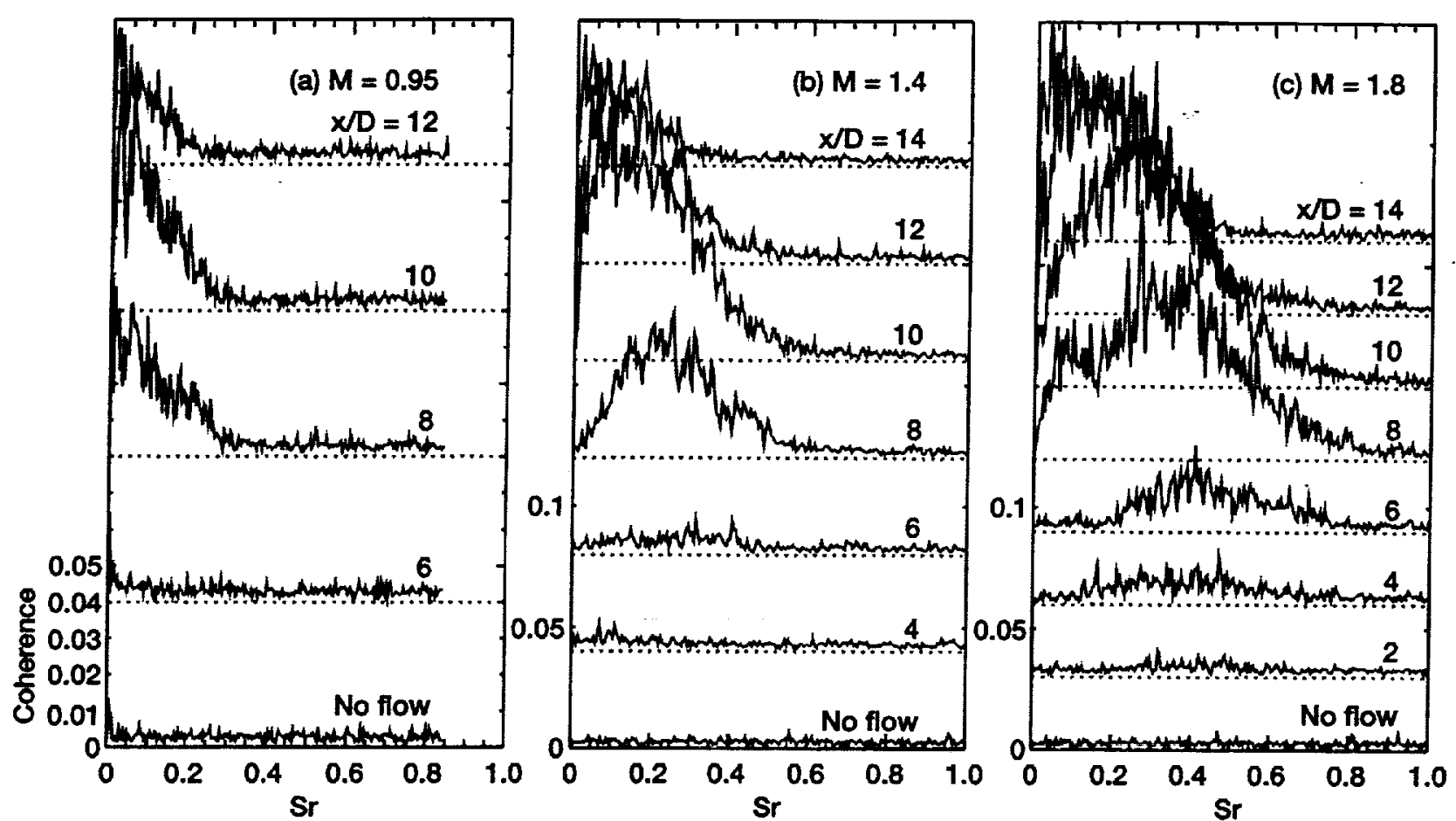

Fig 15. Normalized cross-spectrum between density and sound fluctuations from 3 different jets. The microphone was fixed at $x / D=50, \theta=30^{\circ}$ and the laser position was moved at indicated axial positions along centerline.

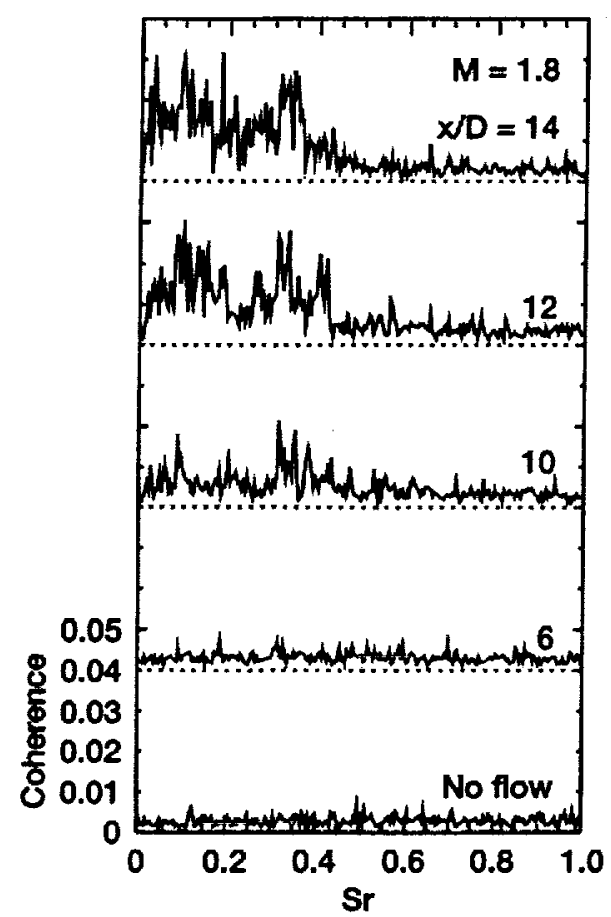

Fig 16. Normalized cross-spectrum between density and sound fluctuations in Mach 1.8 jet. The microphone was fixed at $x / D=50, \theta=90^{\circ}$ and the laser position was moved at indicated axial positions along centerline. 

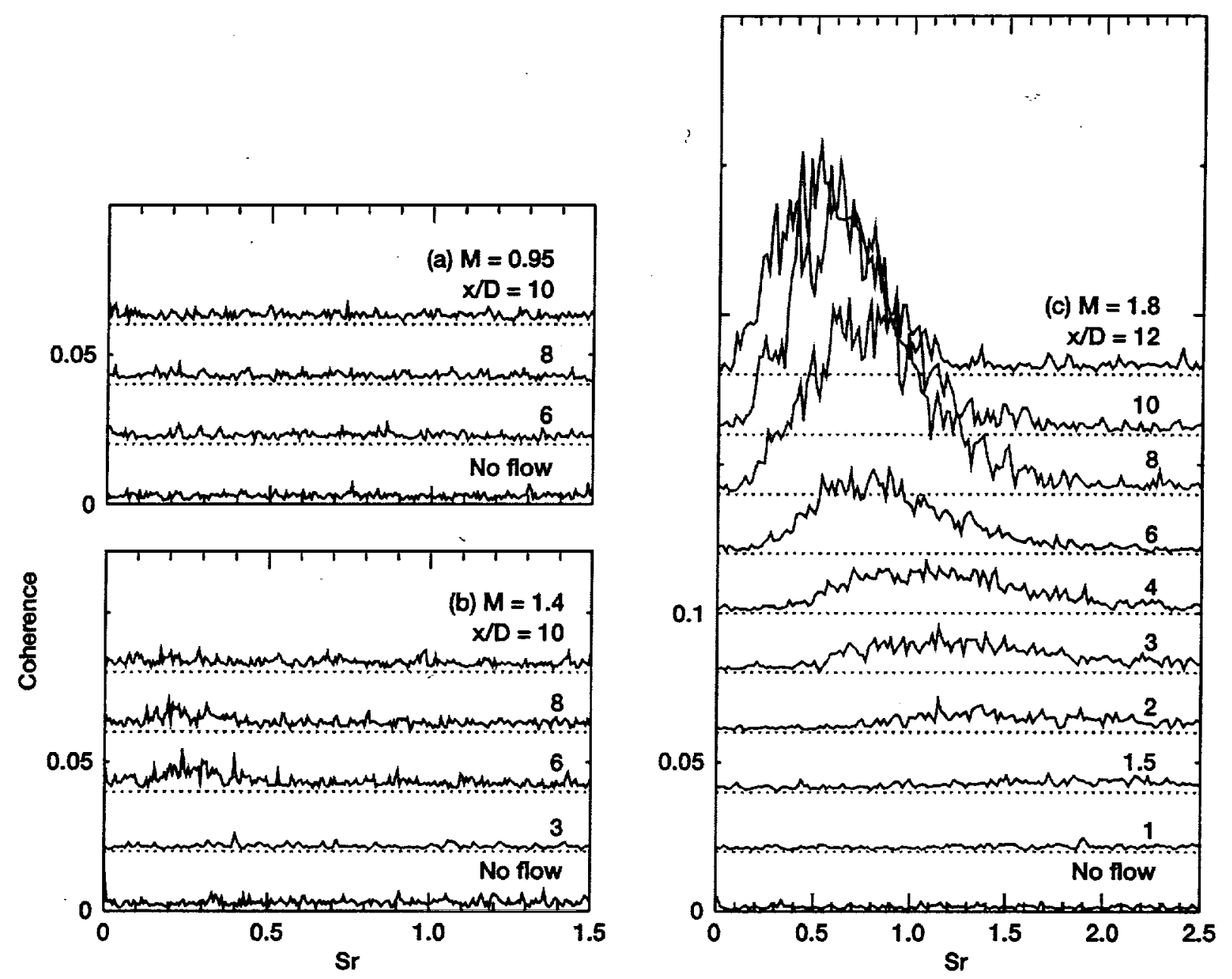

Fig 17. Normalized cross-spectrum between density and sound fluctuations from 3 different jets. The microphone was fixed at $x / D=50, \theta=30^{\circ}$ and the laser position was moved at indicated axial positions along shear layer, $y / D=0.45$. 
